\title{
High Range Resolution Ultrasonographic Vascular Imaging Using Frequency Domain Interferometry With the Capon Method
}

\author{
Hirofumi Taki*, Member, IEEE, Kousuke Taki, Takuya Sakamoto, Member, IEEE, \\ Makoto Yamakawa, Member, IEEE, Tsuyoshi Shiina, Member, IEEE, Motoi Kudo, and
}

Toru Sato, Member, IEEE

\begin{abstract}
For high range resolution ultrasonographic vascular imaging, we apply frequency domain interferometry with the Capon method to a single frame of in-phase and quadrature (IQ) data acquired using a commercial ultrasonographic device with a $7.5 \mathrm{MHz}$ linear array probe. In order to tailor the adaptive beamforming algorithm for ultrasonography we employ four techniques: frequency averaging, whitening, radio-frequency data oversampling, and the moving average. The proposed method had a range resolution of $0.05 \mathrm{~mm}$ in an ideal condition, and experimentally detected the boundary couple $0.17 \mathrm{~mm}$ apart, where the boundary couple was indistinguishable from a single boundary utilizing a B-mode image. Further, this algorithm could depict a swine femoral artery with a range beam width of 0.054 $\mathrm{mm}$ and an estimation error for the vessel wall thickness of 0.009 mm, whereas using a conventional method the range beam width and estimation error were 0.182 and $0.021 \mathrm{~mm}$, respectively. The proposed method requires $7.7 \mathrm{~s}$ on a mobile $P C$ with a single CPU for a $1 \times 3 \mathrm{~cm}$ region of interest. These findings indicate the potential of the proposed method for the improvement of range resolution in ultrasonography without deterioration in temporal resolution, resulting in enhanced detection of vessel stenosis.
\end{abstract}

Index Terms-Adaptive signal processing, Capon method, frequency domain interferometry, ultrasonic imaging, vascular ultrasound.

\section{INTRODUCTION}

$\mathbf{E}$ ARLY and accurate detection of artery stenosis is important for early diagnosis and treatment of lifestyle diseases. Since ultrasonography (US) is one of the primary imaging modalities for the investigation of artery stenosis [1]-[5], im-

Manuscript received August 03, 2011; revised September 22, 2011; accepted September 22, 2011. Date of publication October 06, 2011; date of current version February 03, 2012. This work was supported in part by the Innovative Techno-Hub for Integrated Medical Bio-imaging Project of the Special Coordination Funds for Promoting Science and Technology, from the Ministry of Education, Culture, Sports, Science and Technology (MEXT), Japan. Asterisk indicate corresponding author.

${ }^{*} \mathrm{H}$. Taki is with the Graduate School of Informatics, Kyoto University, Yoshida-honmachi, Sakyo-ku, Kyoto 606-8501, Japan (e-mail: hirofumi.taki@mb6.seikyou.ne.jp).

T. Sakamoto and T. Sato are with the Graduate School of Informatics, Kyoto University, Yoshida-honmachi, Sakyo-ku, Kyoto 606-8501, Japan (e-mail: t-sakamo@i.kyoto-u.ac.jp; tsato@kuee.kyoto-u.ac.jp).

K. Taki and M. Kudo are with the Department of Anatomy, Shiga University of Medical Science, Seta Tsukinowa-cho, Otsu City, Shiga 520-2192, Japan (e-mail: taki@belle.shiga-med.ac.jp; kudo@belle.shiga-med.ac.jp).

M. Yamakawa is with the Advanced Biomedical Engineering Research Unit, Kyoto University, Yoshida-honmachi, Sakyo-ku, Kyoto 606-8501, Japan (e-mail: m.yamakawa@kt8.ecs.kyoto-u.ac.jp).

T. Shiina is with the Graduate School of Medicine, Kyoto University, Yoshida-honmachi, Sakyo-ku, Kyoto 606-8501, Japan (e-mail: shiina@hs.med.kyoto-u.ac.jp).

Color versions of one or more of the figures in this paper are available online at $\mathrm{http}: / /$ ieeexplore.ieee.org.

Digital Object Identifier 10.1109/TMI.2011.2170847 provement in range resolution is highly desirable. Frequency domain interferometry (FDI) imaging methods using two frequencies have been proposed for the improvement of range resolution [6]-[8]. These methods are commonly applied in radar signal processing. However, because only two frequencies are used the results are ambiguous, and unsuitable for an environment with multiple targets [9]. For further improvement of range resolution in radar signal processing, an FDI imaging method using multiple frequencies has been proposed [10]. This method employs adaptive beamforming algorithms to improve range resolution, using multiple frequencies of narrow-band signals. Therefore, blind application of this method to US is unlikely to be successful.

Since the 1960s, adaptive beamforming algorithms have been developed to achieve high spatial resolution and suppress undesired signal contribution. One of the most common approaches calculates a set of weights by minimizing the output power, subject to the constraint that a desired signal gives a constant response [11], [12]. In US, a desired signal and interferences are strongly correlated, and thus the power minimizing method tends to cancel the desired signal using the interferences [13], [14]. Since the spatial averaging technique has been employed to decorrelate a desired signal and an interference signal [15]-[18], several groups have applied the Capon method with spatial averaging to US [19]-[22]. However, these studies were directed at improving the lateral resolution of US. Another series of approaches employed eigenvalue adaptive beamforming algorithms [23]-[28]. These methods introduced an assumption that targets are modeled as points, and thus the size of the sampling grid should be small enough to provide sufficient robustness and spatial resolution. The application of these methods in a clinical environment is extremely difficult, because they involve enormous computational costs. In addition, they need to be utilized under the condition of a high signal-to-noise ratio (SNR).

In previous reports, we presented a high range resolution method based on FDI with an adaptive beamforming algorithm [29], [30]. In the present study, we present a high range resolution method employing four techniques suitable for US: frequency averaging, whitening, RF data oversampling, and moving average. We explain the method, present experimental results obtained utilizing a commercial US device, and offer a conclusion related to our findings.

\section{MATERIALS AND METHODS}

The presented method is based on FDI with the Capon method, an adaptive beamforming algorithm. For the improve- 


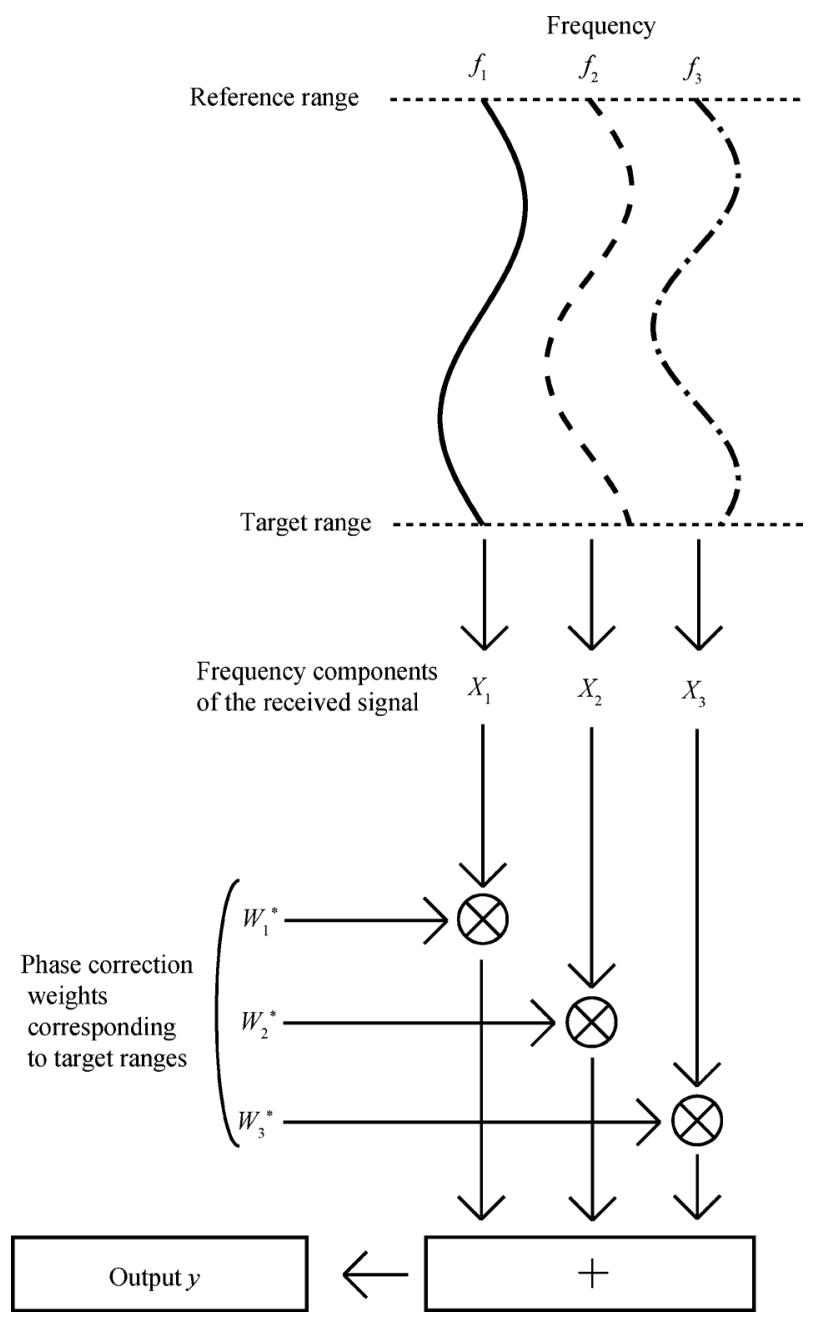

Fig. 1. Schema of the range beamforming of a FDI imager using a weighting vector for phase compensation.

ment of range resolution in US, we apply this method to each individual scan line of in-phase and quadrature (IQ) data. In this section, we briefly describe FDI and the Capon method, and subsequently explain the four techniques employed to tailor the method to US.

\section{A. Data Processing Method Based on FDI}

FDI is a technique employed to estimate target range and its scattering cross section, using the phases and intensities of the frequency components. The phase of each frequency component of a received signal depends on the product of the frequency and the target range, as shown in Fig. 1. Therefore, the summation of the frequency components of a received signal after phase compensation emphasizes the echo returned from a desired range. The output of a FDI imager is given by

$$
\begin{aligned}
y & =\mathbf{X}^{\mathrm{T}} \mathbf{W}^{*} \\
\mathbf{X} & =\left[\begin{array}{llll}
X_{1} & X_{2} & \cdots & X_{N}
\end{array}\right]^{\mathrm{T}} \\
\mathbf{W} & =\left[\begin{array}{llll}
W_{1} & W_{2} & \cdots & W_{N}
\end{array}\right]^{\mathrm{T}}
\end{aligned}
$$

where $\mathbf{W}$ is a weighting vector, $N$ is the number of frequency component samples used for imaging, $\mathbf{X}$ is a set of frequency components of the signal cut out from the received signal of a scan line in a single frame using a window function after a common delay and sum process, and [ $]^{*}$ and []$^{\mathrm{T}}$ denote the complex conjugate and the transpose, respectively. The output power of a FDI imager is

$$
\begin{aligned}
p & =y y^{*}=\mathbf{W}^{\mathrm{T}^{*}} \mathbf{R W} \\
\mathbf{R} & =\mathbf{X X}^{\mathrm{T}^{*}}
\end{aligned}
$$

where []$^{\mathrm{T} *}$ denotes the conjugate transpose. The beamformer method calculates the estimated intensity at a measurement range using a fixed weighting vector $\mathbf{W}=\mathbf{C}$, where

$$
\mathbf{C}=\left[\begin{array}{llll}
e^{\mathrm{j} k_{1} r} & e^{\mathrm{j} k_{2} r} & \cdots & e^{\mathrm{j} k_{N} r}
\end{array}\right]^{\mathrm{T}} .
$$

$k_{l}$ is the $l$ th wavenumber of the frequency components of the received signal, and $r / 2$ is the measurement range.

\section{B. Capon Method}

When multiple targets exist within a range gate, the range resolution of the beamformer method deteriorates due to the interference of targets that exist near the measurement range. Thus, we employ the Capon method, minimizing the contribution from other ranges subject to a constant response at a desired range [11]. This problem is expressed as follows:

$$
\text { minimize } \quad P(r) \quad \text { subject to } \quad \mathbf{C}^{\mathrm{T}^{*}} \mathbf{W}=1 \text {. }
$$

This problem can be solved by the application of Lagrange multiplier methods. The solution to (7) is given by

$$
P_{\text {Cap }}(r)=\frac{1}{\mathbf{C}^{\mathrm{T}^{*}} \mathbf{R}^{-1} \mathbf{C}}
$$

where $\mathbf{R}^{-1}$ denotes the inverse matrix of $\mathbf{R} \cdot P_{\text {Cap }}(r)$ denotes the Capon range profile [31]. The Capon method can estimate the ranges of multiple targets when the number of targets is less than that of the size of $\mathbf{R}$ [18], indicating the possibility of a FDI imager with the Capon method in a clinical application.

\section{RF Data Oversampling}

US devices utilize quadrature detectors to acquire IQ data. The detection is equivalent to the multiplication processes between a received signal and two sinusoidal waves, where the phase difference of the two sinusoidal waves is $90^{\circ}$ and their center frequency is equal to the transmit center frequency. When a target exists at the range $r / 2$, the received RF data signal is given by

$$
S(t)=S^{\prime}\left(t-\frac{r}{c}\right)=\sum_{k} a_{k} \cos \left\{\omega_{k}\left(t-\frac{r}{c}\right)+\theta_{k}\right\}
$$

where $S(t)$ is a received RF data signal with a common delay and sum process in the time domain before quadrature detection, $S^{\prime}(t)$ is the reference RF data signal when the same target exists at the origin, $c$ is the velocity of the ultrasound, $a_{k} \cos \left(\omega_{k} t+\theta_{k}\right)$ is the frequency component of $S^{\prime}(t)$ at the $k$ th angular frequency $\omega_{k}$ and $a_{k}$ and $\theta_{k}$ are real numbers. Here, we account only the time delay caused by the transmission of the path $r$. The sampling time of each quadrature data is a quarter period of the base sinusoid signal later than that of the corresponding in-phase 
data. The received IQ data signal after mixing (i.e., multiplication with base sinusoid signals and low-pass filtering) is

$$
\begin{aligned}
& S_{\mathrm{IQ}}(n \Delta T)=S_{\mathrm{I}}(n \Delta T)+\mathrm{j} S_{\mathrm{Q}}(n \Delta T) \\
& S_{\mathrm{I}}(n \Delta T) \cong \mathrm{LPF}\left[S^{\prime}\left(n \Delta T-\frac{r}{c}\right) \cos \left(\omega_{\mathrm{s}} n \Delta T\right)\right] \\
\cong & \frac{1}{2} \sum_{k} a_{k} \cos \left\{\left(\omega_{k}-\omega_{\mathrm{s}}\right) n \Delta T-\frac{r \omega_{k}}{c}+\theta_{k}\right\} \\
= & \frac{1}{4} \sum_{k} a_{k}\left\{\operatorname{expj}\left(\theta_{k}^{\prime}-\frac{r \omega_{k}}{c}\right)+\operatorname{expj}\left(-\theta_{k}^{\prime}+\frac{r \omega_{k}}{c}\right)\right\} \quad(11) \\
& S_{\mathrm{Q}}(n \Delta T) \\
\cong & \mathrm{LPF}\left[S^{\prime}\left(n \Delta T-\frac{r}{c}+\frac{T_{\mathrm{S}}}{4}\right) \sin \left\{\omega_{\mathrm{s}}\left(n \Delta T+\frac{T_{\mathrm{S}}}{4}\right)\right\}\right] \\
\cong & \frac{1}{2} \sum_{k} a_{k} \sin \left\{\left(\omega_{k}-\omega_{\mathrm{s}}\right)\left(n \Delta T+\frac{T_{\mathrm{S}}}{4}\right)-\frac{r \omega_{k}}{c}+\theta_{k}\right\} \\
= & \frac{1}{4 \mathrm{j}} \sum_{k} a_{k}\left\{\operatorname{expj}\left(\theta_{k}^{\prime}+\theta_{k}^{\prime \prime}-\frac{r \omega_{k}}{c}\right)-\operatorname{expj}\left(-\theta_{k}^{\prime}-\theta_{k}^{\prime \prime}+\frac{r \omega_{k}}{c}\right)\right\} \\
& \theta_{k}^{\prime}=\left(\omega_{k}-\omega_{\mathrm{s}}\right) n \Delta T+\theta_{k} \\
& \theta_{k}^{\prime \prime}=\left(\omega_{k}-\omega_{\mathrm{s}}\right) \frac{T_{\mathrm{S}}}{4}
\end{aligned}
$$

where $S_{\mathrm{I}}(n \Delta T)$ and $S_{\mathrm{Q}}(n \Delta T)$ are the $n$th signal of the in-phase and quadrature data, respectively, LPF[] denotes the low pass filtering, $\Delta T$ is the sampling interval of quadrature detection, and $\omega_{s}$ and $T_{\mathrm{S}}$ are the angular frequency and the period at the transmit center frequency, respectively. When continuous wave Doppler is utilized, $\omega_{k}$ is approximately equal to $\omega_{s}$. Therefore, the phase gap of each frequency component caused by the target range is relative to the product of the frequency and the target range. However, pulse wave Doppler uses broadband signals, and the waveform of the IQ data signal returned from the range $r / 2$ is different from that of the reference IQ data signal returned from the target at the origin

$$
\begin{aligned}
S_{\mathrm{IQ}}^{\prime}(n \Delta T)= & \frac{1}{2} \sum_{k} a_{k}\left\{\cos \left(\theta_{k}^{\prime}\right)+\mathrm{j} \sin \left(\theta_{k}^{\prime}+\theta_{k}^{\prime \prime}\right)\right\} \\
S_{\mathrm{IQ}}^{\prime \prime}(n \Delta T)= & \frac{1}{4} \sum_{k} a_{k}\left\{b_{k r} \operatorname{expj}\left(\theta_{k}^{\prime}-\frac{r \omega_{k}}{c}\right)\right. \\
& \left.+b_{k r}^{\prime} \operatorname{expj}\left(-\theta_{k}^{\prime}+\frac{r \omega_{k}}{c}\right)\right\} \\
& +\frac{1}{4} \sum_{k} a_{k}\left\{b_{k r} \operatorname{expj}\left(\theta_{k}^{\prime}+\theta_{k}^{\prime \prime}-\frac{r \omega_{k}}{c}\right)\right. \\
= & \frac{1}{2} \sum_{k} a_{k}\left\{\cos \left(\theta_{k r}^{\prime}-\frac{r \omega_{s}}{c}\right)\right. \\
b_{k r}= & \operatorname{expj}\left\{\frac{r\left(\omega_{k}-\omega_{s}^{\prime}\right)}{c}\right\} \\
b_{k r}^{\prime}= & \operatorname{expj}\left\{\frac{-r\left(\omega_{k}-\omega_{s}^{\prime \prime}\right)}{c}\right\}
\end{aligned}
$$

where $S_{\mathrm{IQ}}^{\prime}(n \Delta T)$ is the reference IQ data signal, $S_{\mathrm{IQ}}$ " $(n \Delta T)$ is the IQ data signal after range compensation and $b_{k r}$ and $b_{k r}$ ' are

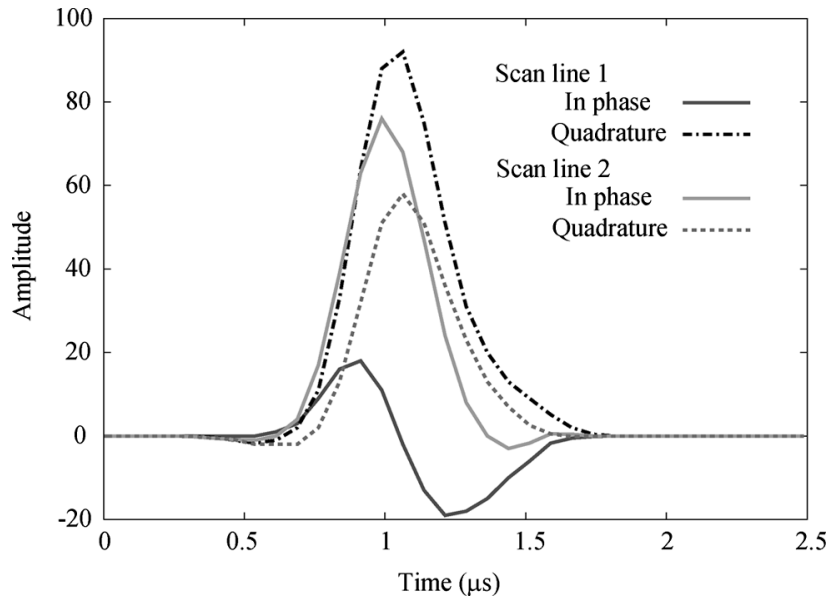

Fig. 2. Echoes of IQ data returned from a boundary between $20 \%$ gelatin and $4 \%$ agar in two scan lines, where the transmit center frequency and the sampling frequency of the quadrature detection are 7.5 and $15 \mathrm{MHz}$, respectively.

range compensation values. Both waveforms corresponds with each other under the condition

$$
\frac{r \omega_{s}}{c}=2 \pi
$$

Since the waveform of a received signal after quadrature detection depends on the phase difference between the received signal and sinusoidal waves, the echo waveform of IQ data returned from a target is often different than that from the same target, as shown in Fig. 2. FDI imaging method assumes that the received signal consists of multiple echoes of targets and noise, where the waveform of each echo is similar to that of the reference signal. Therefore, blind application of FDI to a received IQ data signal is unlikely to be successful.

We introduce the assumption that the IQ data signal is equal to the product of the RF data signal and the sinusoidal waves for quadrature detection

$$
\begin{aligned}
S_{\mathrm{I}}(n \Delta T) & \cong S(n \Delta T) \cos \left(\omega_{\mathrm{s}} n \Delta T\right) \\
S_{\mathrm{Q}}(n \Delta T) & \cong S\left(n \Delta T+\frac{T_{\mathrm{S}}}{4}\right) \sin \left(\omega_{\mathrm{s}} n \Delta T+\frac{T_{\mathrm{S}}}{4}\right) .
\end{aligned}
$$

In the present study, we propose a technique to reconstruct oversampled received RF data from signals of IQ data

$$
\begin{aligned}
S_{\mathrm{P}}(n \Delta T) & =\frac{S_{\mathrm{I}}(n)}{\cos \left(\omega_{\mathrm{s}} n \Delta T\right)} \\
S_{\mathrm{P}}\left(n \Delta T+\frac{T_{\mathrm{S}}}{4}\right) & =S_{\mathrm{Q}}(n) \sin \left(\omega_{\mathrm{s}} n \Delta T+\frac{T_{\mathrm{S}}}{4}\right)
\end{aligned}
$$

where $S_{\mathrm{P}}(t)$ is the oversampled RF data. We term this technique RF data oversampling. The RF data oversampling process only converts the arrangement of the data set after the amplitude compensation caused by the sinusoidal waves at the quadrature detection.

In our study, $T_{\mathrm{S}}$ is equal to $2 \Delta T$, i.e., the sampling frequency of the quadrature detection is equal to twice the transmit center frequency. In this case, the sampling frequency of the oversampled RF data is four times the transmit center frequency. Fig. 3 shows the oversampled RF data of two scan lines, where the 


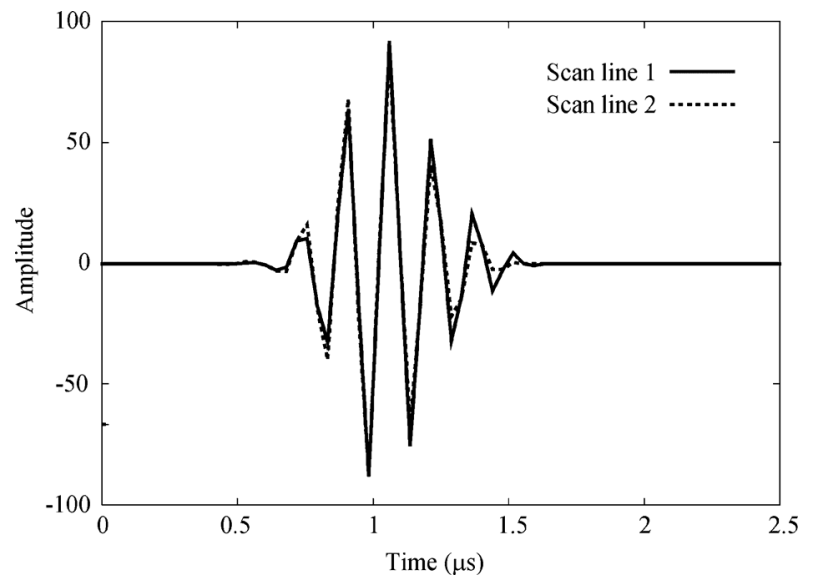

Fig. 3. Echoes of oversampled real data converted from the echoes of IQ data returned from a boundary between gelatin and agar in two scan lines. The peak positions of the envelopes of two echoes are shifted to $1 \mu$ s after amplitude adjustment.

peak positions of the envelopes of scan lines 1 and 2 were adjusted to $1 \mu \mathrm{s}$ using a time shift in the frequency domain after the adjustment of the envelope amplitudes. The time shift process consists of three steps. First, Fourier transform is applied to each scan line. Next, we calculate the phase shift coefficient for the time shift, $p_{\mathrm{Tmin}} \cdot p_{\mathrm{Tmin}}$ is equal to $p_{\mathrm{T}}$ when $p_{\mathrm{D}}$ is minimized

$$
p_{\mathrm{D}}=\sum_{l}\left\{\arg \left(X_{l}\right)-p_{\mathrm{T}} f_{l}\right\}^{2}
$$

where $f_{l}$ is the frequency of the frequency component $X_{l}$. The phase compensation value of each frequency component is the product of the phase shift coefficient and its frequency. After this compensation to all frequency components, inverse Fourier transform is applied to acquire a scan line in the time domain after the time shift. These three steps shifted the peak positions of the envelopes of scan lines 1 and 2 to $1 \mu \mathrm{s}$. This result shows that signals of IQ data were converted to those of oversampled RF data that resembled the received RF data, as shown in Fig. 3. The oversampling factor is $2 f_{s} / B$, where $f_{s}$ is the transmit center frequency, and $B$ is the bandwidth of the transmit pulse. In the present study, $f_{s}=7.5 \mathrm{MHz}$ and the $-6 \mathrm{~dB}$ bandwidth of the signal returned from a flat boundary was $2.4 \mathrm{MHz}$. Therefore, the oversampling factor was 6.25.

\section{Whitening Frequency Components}

Since the Capon method is designed for cases using narrowband signals, it assumes that the frequency components of a signal returned from a target have the same intensity. For the application to US using broadband signals, we apply the whitening technique to the received signals prior to the application of FDI with the Capon method. This technique assumes that the echo waveform return from each target is same as that of a reference signal. This technique corrects the intensity of all frequency components uniformly, using a reference signal returned from a single boundary

$$
X_{\mathrm{H} k}=\frac{X_{\mathrm{P} k} X_{\mathrm{R} k}^{*}}{\left(\left|X_{\mathrm{R} k}\right|^{2}+\eta\right)}
$$

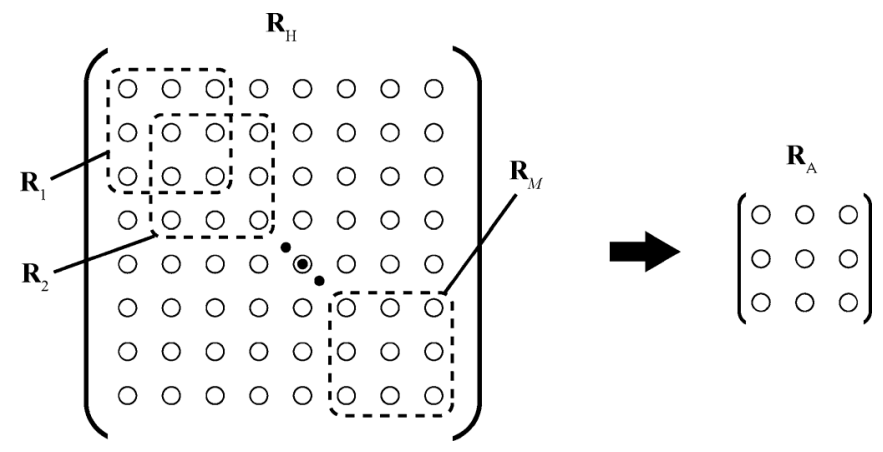

Fig. 4. The construction of the sub-matrix $\mathbf{R}_{\mathrm{A}}$ from a covariance matrix after whitening, $\mathbf{R}_{\mathrm{H}}$.

where $\eta$ is a constant term for stabilization, and $X_{\mathrm{H} k}, X_{\mathrm{P} k}$, and $X_{\mathrm{R} k}$ are $k$ th frequency component of a signal of oversampled RF data after whitening, $k$ th frequency component of a signal of oversampled RF data before whitening, and $k$ th frequency component of a reference signal of a oversampled RF data without whitening, respectively.

Since the optimum filter is the Wiener filter, the optimum value of $\eta$ is equal to the noise intensity of the $k$ th frequency component of the received signal e.g., [32], [33]. The noise intensity of a frequency component can be estimated from the variance expectation of the intensity of the component when the signal intensity is constant. However, the signal intensity is commonly variable in ultrasound imaging, especially in imaging involving fast moving organs such as an artery or the heart. As a consequence, we introduce the assumption that the expectation of the noise intensity is uniform in all frequency components, and employ the average intensity of the frequency components where the components are supposed to include slight signal intensity. In our study, we employed the average intensity of the frequency components from 11 to $14 \mathrm{MHz}$ as the value of $\eta$.

\section{E. Capon Method With Frequency Averaging}

FDI applications do not work when the signals received from different targets are correlated. In atmospheric radar signal processing, temporal averaging is applied to the covariance matrix $\mathbf{R}$ to suppress the correlation between echoes from different targets [15]-[18], whereas the environment of common US applications is nonstationary, resulting in the difficulty of applying temporal averaging to $\mathbf{R}$. Thus, we employ the frequency averaging technique to decorrelate signals from different targets

$$
\begin{aligned}
\mathbf{R}_{\mathrm{A}} & =\frac{1}{M} \sum_{m=1}^{M} \mathbf{R}_{m} \\
R_{m i j} & =X_{\mathrm{H}(i+m-1)} X_{\mathrm{H}(j+m-1)}^{*}
\end{aligned}
$$

where $\mathbf{R}_{\mathrm{A}}$ is a covariance matrix of a received signal after frequency averaging and $R_{m i j}$ is the $(i, j)$ element of a $m$ th submatrix $\mathbf{R}_{m}$ and $X_{\mathrm{H}}$ is the oversampled RF data converted from a received signal of IQ data after whitening. Each matrix $\mathbf{R}_{m}$ is extracted from the same whitened full-matrix $\mathbf{R}_{\mathrm{H}}$ to construct the sub-matrix $\mathbf{R}_{\mathrm{A}}$, as shown in Fig. 4. This technique requires 
that the sampling frequency interval is constant. By the employment of whitening and frequency averaging, the estimated intensity expressed in (4) is modified to

$$
\begin{aligned}
P^{\prime} & =\mathbf{W}_{\mathrm{A}}^{\mathrm{T}^{*}} \mathbf{R}_{\mathrm{A}} \mathbf{W}_{\mathrm{A}} \\
\mathbf{W}_{\mathrm{A}} & =\left[\begin{array}{llll}
W_{1}^{\prime} & W_{2}^{\prime} & \cdots & W_{n-m+1}^{\prime}
\end{array}\right]^{\mathrm{T}} .
\end{aligned}
$$

Consequently, the minimization of the contribution of signals from undesired ranges subject to a constant response at a desired range is modified to

$$
\begin{aligned}
& \min \mathbf{W}_{\mathrm{A}}^{\mathrm{T}^{*}} \mathbf{R}_{\mathrm{A}} \mathbf{W}_{\mathrm{A}} \text { subject to } \mathbf{C}_{\mathrm{A}}^{\mathrm{T}^{*}} \mathbf{W}_{\mathrm{A}}=1 \\
& \mathbf{C}_{\mathrm{A}}=\left[\begin{array}{lll}
\exp \left(\mathrm{j} k_{1} r\right) & \cdots & \exp \left\{\mathrm{j} k_{(N-M+1)} r\right\}
\end{array}\right]^{\mathrm{T}} \text {. }
\end{aligned}
$$

The solution to (30) and (31) is given by

$$
P_{\mathrm{Cap}}^{\prime}(r)=\frac{1}{\mathbf{C}_{\mathrm{A}}^{\mathrm{T}^{*}}\left(\mathbf{R}_{\mathrm{A}}+\eta^{\prime} \mathbf{E}\right)^{-1} \mathbf{C}_{\mathrm{A}}}
$$

where $\eta^{\prime} \mathbf{E}$ is a diagonal loading matrix to obtain the inverse matrix $\mathbf{R}_{\mathrm{A}}^{-1}$ stably. Since the proposed method is one of adaptive beamforming techniques, the values of the weighting function vary in order to adapt the condition of the received signal. The employment of the optimum weighting function gives the estimated intensity expressed by (32). Thus, it is not necessary to acquire the weighting vector itself for each desired range. The addition of $\eta^{\prime} \mathrm{E}$ is equivalent to the addition of white noise to the received signal. This process suppresses the SNR, causing the deterioration of the range resolution of the FDI imaging method [31]. Therefore, we should employ a small $\eta$ ' to stabilize $\mathbf{R}_{\mathrm{A}}^{-1}$ and to maintain the image quality. In the present study, we employed $-40 \mathrm{~dB}$ of the average intensity of the received signal in the range between 5 to $9 \mathrm{MHz}$ as the value of $\eta$ '. We apply the FDI method on a line-by-line basis. Therefore, the covariance matrix $\mathbf{R}$, the sub-matrix $\mathbf{R}_{\mathrm{A}}$ and the inverse matrix of $\left(\mathbf{R}_{\mathrm{A}}+\eta^{\prime} \mathbf{E}\right)$ are calculated only once for each scan line.

\section{F. Moving Average in the Lateral Direction}

The proposed method calculates the echo intensities in each scan line separately. Therefore, the suppression level of the correlation between echoes from different targets varies with the individual scan line. Since the estimated intensities largely depend on the suppression level of the correlation between echoes from different targets, the image acquired by the proposed method has lower continuity in the lateral direction than a conventional B-mode image. To improve the lateral continuity of the image acquired by the proposed method, we apply the moving average to the acquired image in the lateral direction. When the frequency averaging technique completely suppresses the correlation between echoes from different targets in all the scan lines, the acquired image spreads in the region that is determined by the point spread function of a US device. Since the spread of the acquired image in the lateral direction is supposed to be equal to the ultrasound beam width, we employ the half-power width of the ultrasound beam of a US device as the width of moving average in the lateral direction. We applied this technique to the imaging of swine femoral arteries.

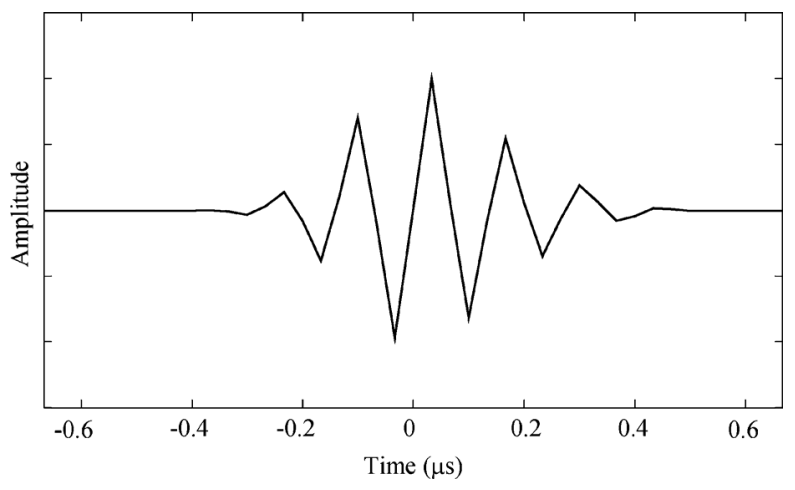

Fig. 5. Waveform of the reference signal used for the proposed imaging method.

\section{G. Experimental Setup}

In this study, we used IQ data acquired by a commercial US device to evaluate the potential of the proposed method for its application to general US devices. Experiments were conducted using a Hitachi EUB-8500 (Hitachi, Tokyo, Japan) US device with a $7.5 \mathrm{MHz}$ linear array probe, which has the function of exporting raw IQ data. The scan line interval and range interval of the US device were about 0.13 and $0.05 \mathrm{~mm}$, respectively. In our study, we supposed the sound velocity $c$ to be $1500 \mathrm{~m} / \mathrm{s}$. The transmit focal range was $2 \mathrm{~cm}$. The $-6 \mathrm{~dB}$ bandwidth of the echo from a single flat boundary between the gelatin and agar was $2.4 \mathrm{MHz}$, where the gelatin layer located forward and the echo waveform is shown in Fig. 5. The half-power width of the ultrasound beam of the US device in the lateral direction was about $0.6 \mathrm{~mm}$ at a depth of $2 \mathrm{~cm}$. For the investigation of the range resolution using the proposed method, we prepared three $20 \%$ gelatin blocks. Each block had an agar membrane at a depth of $2 \mathrm{~cm}$, and the thicknesses of the three membranes were $0.17,0.25$, and $0.33 \mathrm{~mm}$, respectively. We first prepared three $4 \%$ agar membranes, and then embedded them into the gelatin blocks after airing. We employed the airing process to compress the thickness of the membrane because it was difficult to prepare a flat membrane of a thickness less than $0.3 \mathrm{~mm}$. Following ultrasound imaging an anatomist immediately measured the actual thickness of each agar membrane. Measurements were conducted with microscopic observation of the vertical section through each gelatin block containing the agar membrane. Fig. 6 shows the microscopic images of the agar membranes. Throughout the experiments we utilized the echo from a boundary between $20 \%$ gelatin and $4 \%$ agar as a reference signal, as shown in Fig. 5.

To examine the potential of the proposed method for clinical applications we further applied this method to a fresh swine femoral artery, as shown in Fig. 7. In addition, we used microscopy to compare the performance of the proposed method to that of the conventional method in the estimation of vessel wall thickness. For this comparison we prepared a gelatin block containing a swine femoral artery as follows. We immersed the swine femoral artery in $4 \%$ formaldehyde $0.1 \mathrm{M}$ sodium phosphate buffer $(\mathrm{pH} 7.0)$ overnight at room temperature. After this fixation process the artery was preserved in $50 \mathrm{mM}$ phosphate-buffered $0.9 \%$ saline (PBS) containing $0.02 \% \mathrm{NaN}_{3}$ to stop the fixation reaction. Then the artery was embedded in a 

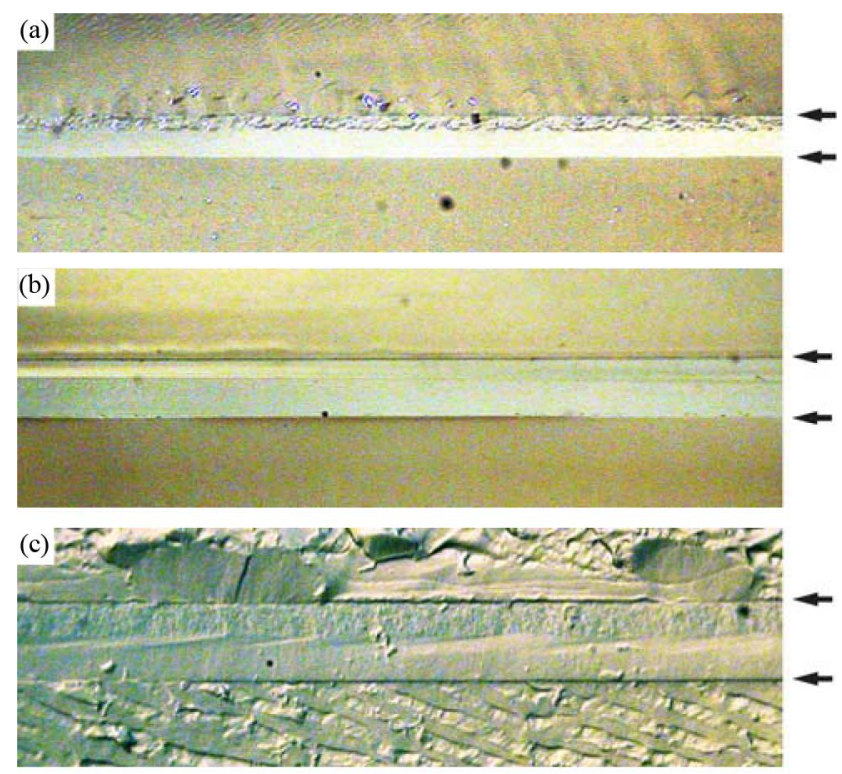

(d)

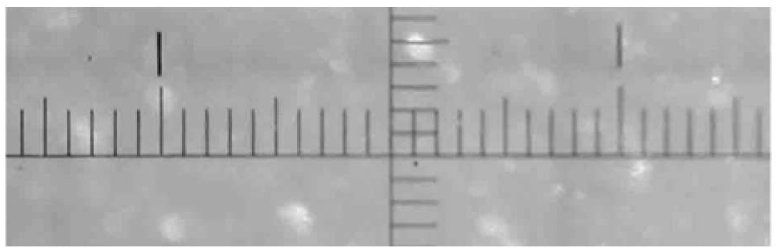

Fig. 6. Microscopic images of agar membranes (a) 0.17 (b) 0.25 , and (c) 0.33 $\mathrm{mm}$ thick, where an anatomist had enhanced the contrast of agar membrane images. Arrows indicate the boundaries between the agar membranes and gelatin. (d) Microscopic image of a ruler with a millimeter scale.

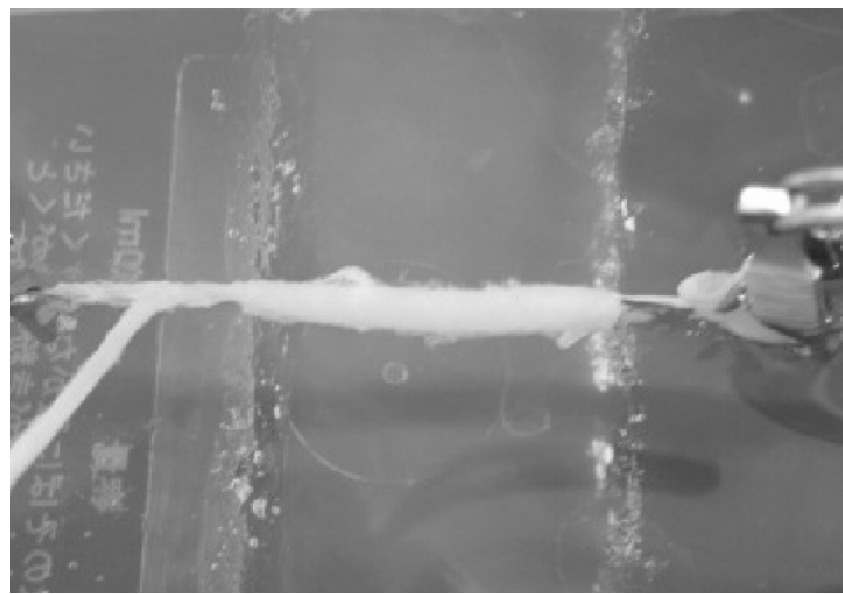

Fig. 7. Fresh swine femoral artery utilized in the study.

$20 \%$ gelatin block. The gelatin block containing the artery then underwent further fixation in $4 \%$ formaldehyde in $0.1 \mathrm{M}$ sodium phosphate buffer for one day at room temperature. After the second fixation process, the block was preserved in $50 \mathrm{mM}$ PBS containing $0.02 \% \mathrm{NaN}_{3}$ for one day. First, we settled the gelatin block with the artery in a cutting case, as shown in Fig. 8. The longitudinal axis of the artery was adjusted with the cutting slit pair, where the slit pair were also utilized as the position marker at ultrasound imaging. After ultrasound imaging, an anatomist cut the gelatin block with a blade inserted into the slit pair. This

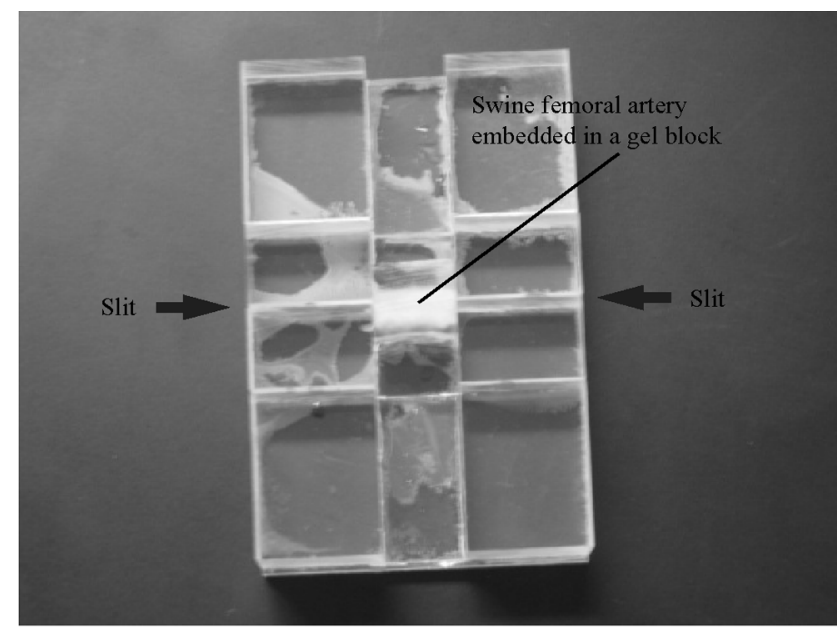

(a)

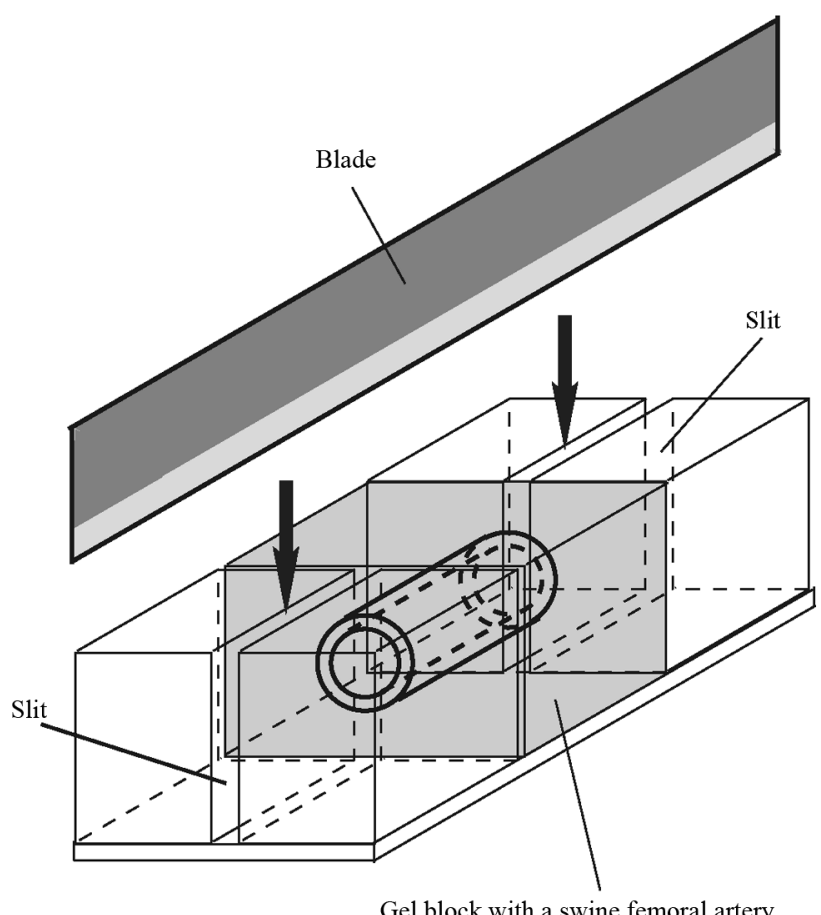

Gel block with a swine femoral artery

(b)

Fig. 8. Microscopic investigation of the ultrasound measurement plane. (a) We acquired the ultrasound image at the section that included the slits of the cutting case. (b) Next, an anatomist inserted a blade in the slits for microscopic investigation.

process guaranteed that the cut site was very close to the ultrasound-imaging site. The section of the gelatin block was photographed microscopically in order to measure the actual thickness of the vessel wall by an anatomist. We used the boundaries traced microscopically to evaluate the performance of the proposed method in the depiction of vessel wall boundaries.

The sampling frequency of the oversampled RF data is 30 $\mathrm{MHz}$, i.e., the range interval is $0.025 \mathrm{~mm}$. Since we cut out the oversampled RF data of $1.5-2.5 \mathrm{~cm}$ in depth using a rectangular window function, the number of range samples is 401 per scan line. We add 599 zeros after the extracted signals in the time domain to reduce the sampling interval in the frequency domain. After the process of zero-padding in the time domain, the mixed 
radix FFT operation [34] is applied to the 1000-point data. The sampling interval in the frequency domain is $30 \mathrm{kHz}$. We utilize the 134 frequency components from 5 to $9 \mathrm{MHz}$ out of 1000 frequency samples.

Since the half-power width of the ultrasound beam of the US device was about $0.6 \mathrm{~mm}$, i.e., about the width of five scan lines, we used five scan lines for the moving average in the lateral direction. We employed the range interval of $0.01 \mathrm{~mm}$ for the proposed method, because the range beam width of the proposed method was supposed to be narrower than the range sampling interval of the received signal. To adjust the range interval of the B-mode image to that of the FDI image, we constructed B-mode images with a range interval of $0.01 \mathrm{~mm}$ using a zero-padding process in the frequency domain. Since the range interval of a B-mode image was $0.05 \mathrm{~mm}$, a B-mode image had 200 range samples in the region of interest (ROI) from 1.5 to $2.5 \mathrm{~cm}$ deep. After the Fourier transform each scan line of the estimated intensity in the time domain was converted to that in the frequency domain, where the frequency components ranged from -7.5 to $7.5 \mathrm{MHz}$ and the number of the components was 200 . We added a couple of 400 zeros at both edges of each scan line of the estimated intensity in the frequency domain. The inverse Fourier transform of the scan line after zero-padding in the frequency domain adjusted the range interval of the B-mode image to $0.01 \mathrm{~mm}$.

\section{RESULTS}

We first investigate the spatial resolution of the proposed method numerically in an ideal condition without noise. We then apply the proposed method to agar membranes and to a swine femoral artery to examine the potential of the proposed method experimentally.

\section{A. Spatial Resolution of the Proposed Method in an Ideal Condition}

We investigate the spatial resolution of the proposed method in an ideal condition where no more than two targets exist in a ROI, each of the echo waveforms returned from the targets is the same as that of the reference signal, and the received signal includes no noise. The echo returned from two targets is given by

$$
S_{\mathrm{IDL}}\left(I_{\mathrm{T}}, t\right)=S_{\mathrm{R}}(t)+S_{\mathrm{R}}\left(t-\frac{2 I_{\mathrm{T}}}{c}\right)
$$

where $I_{\mathrm{T}}$ is the target interval and $S_{\mathrm{R}}(t)$ is the reference signal. The reference signal is the echo from a boundary between $20 \%$ gelatin and 4\% agar, as shown in Fig. 5. Fig. 9 shows the waveforms of the received signal returned from a single target and from a couple of targets, where the target intervals are 0.025 , $0.05,0.075$, and $0.1 \mathrm{~mm}$. Since the $-6 \mathrm{~dB}$ bandwidth of the echo from a target is $2.4 \mathrm{MHz}$, the range resolution of the conventional B-mode image is about $0.31 \mathrm{~mm}$. Therefore, the conventional method using the intensity of the signal can hardly indentify two targets with an interval of less than $0.3 \mathrm{~mm}$.

The proposed method failed to detect a couple of targets that were $0.025 \mathrm{~mm}$ apart from each other, regardless of the sub-

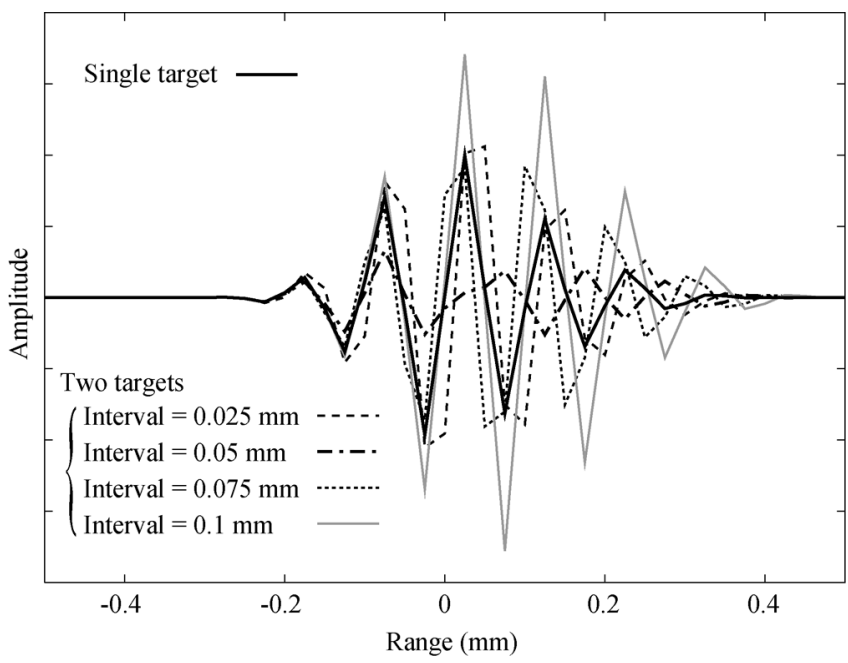

Fig. 9. Waveforms of the received signal returned from a single target and a couple of targets, where the target intervals of the target couples are $0.025,0.05$, 0.075 , and $0.1 \mathrm{~mm}$.

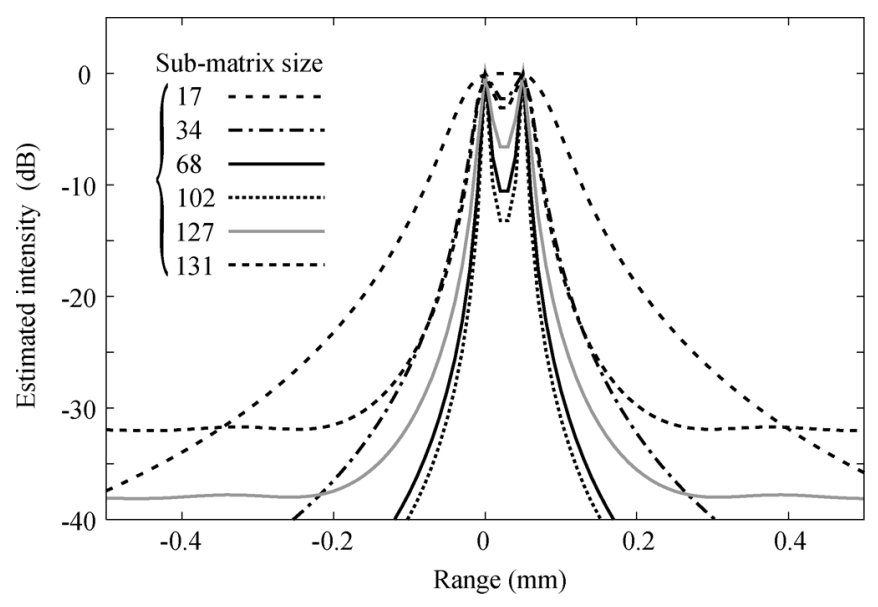

Fig. 10. Estimated intensity of the couple of targets with a target interval of $0.05 \mathrm{~mm}$. The sub-matrix sizes of the proposed method are 17, 34, 68, 102, 127, and 131 , where the size of the covariance matrix after whitening is 134 .

matrix size. We therefore used the case where a couple of targets were $0.05 \mathrm{~mm}$ apart from each other for the investigation of the proper sub-matrix size. Fig. 10 shows the estimated intensity of the couple of targets calculated using the proposed method when the target interval is $0.05 \mathrm{~mm}$. This result shows that the spatial resolution of the proposed method is almost invariable when the sub-matrix size varies from half to $3 / 4$ the size of $\mathbf{R}_{\mathrm{H}}$.

Next, we investigate the computational load of the proposed method. We set the size of a ROI at $1 \times 3 \mathrm{~cm}$, i.e., 1001 range samples $\times 226$ scan lines. A mobile PC with a single CPU (Intel Core2 Duo $2.26 \mathrm{GHz}$ ) and $3 \mathrm{~GB}$ RAM was utilized. The main task in this calculation is the acquisition of the inverse matrix in (32). The number of targets in the ROI does not increase the computational load. Fig. 11 shows the computational load of the proposed method used to calculate the estimated intensity in the ROI. The calculation time of the proposed method increases rapidly as the sub-matrix size enlarged; however, the maximum calculation time for a ROI $1 \times 3 \mathrm{~cm}$ is less than $25 \mathrm{~s}$. 


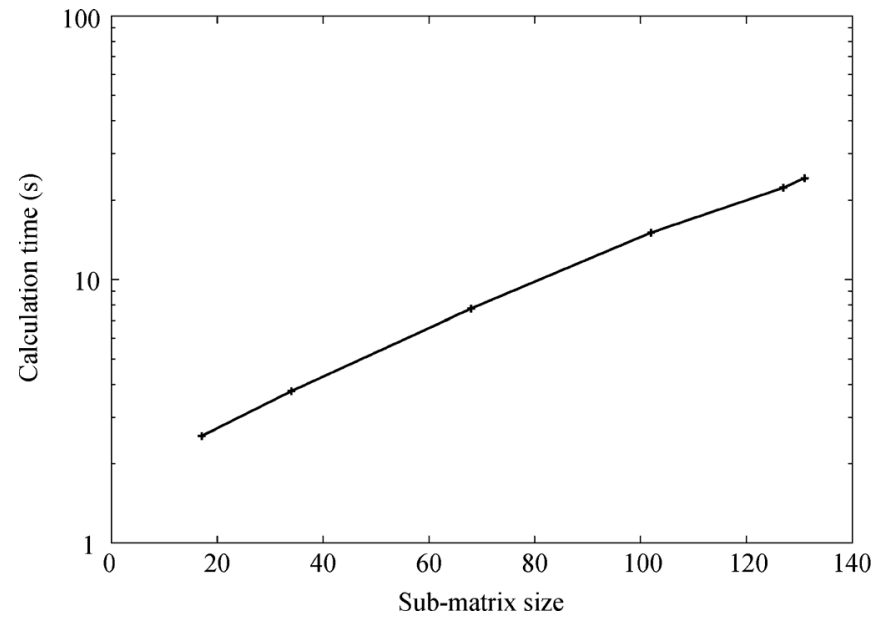

Fig. 11. Calculation time of the proposed method for a ROI of $1 \times 3 \mathrm{~cm}$, i.e., 226 scan lines $\times 1001$ range samples.

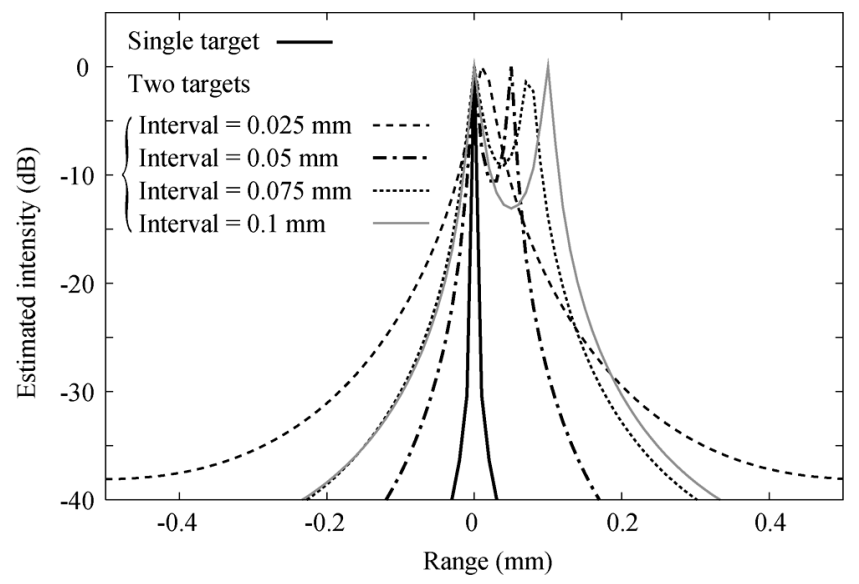

Fig. 12. Estimated intensity of the proposed method with a sub-matrix size of 68. ROI includes a single target or a couple of targets, where the target intervals are $0.025,0.05,0.075$, and $0.1 \mathrm{~mm}$.

We confirmed that the calculation time using experimental data was almost equal to that using simulation data.

For high spatial resolution with a modest computational load, we employ a sub-matrix size of 68 , which is about half the size of $\mathbf{R}_{\mathrm{H}}$. In this case the calculation time for a ROI of $1 \times 3 \mathrm{~cm}$ was 7.7 s. Fig. 12 shows the estimated intensity of the proposed method with a sub-matrix size of 68 , where the ROI included a single target or a couple of targets. When the target interval is $0.075 \mathrm{~mm}$, the posterior target is located at the median of the two range sample points of 0.07 and $0.08 \mathrm{~mm}$. This result indicates that the proposed method has the spatial resolution of $0.05 \mathrm{~mm}$, and a range interval of $0.01 \mathrm{~mm}$ is sufficient for the proposed method. Thus, we employ a range interval of $0.01 \mathrm{~mm}$ for the proposed method.

\section{B. Agar Phantom Images Using the Proposed Method}

We utilize agar membranes to compare the range resolution of the FDI image and that of the B-mode image. We adjusted the range interval of the B-mode image to that of the FDI image, i.e., both images employed a range interval of $0.01 \mathrm{~mm}$. Fig. 13 shows a conventional B-mode image of a gelatin block with an

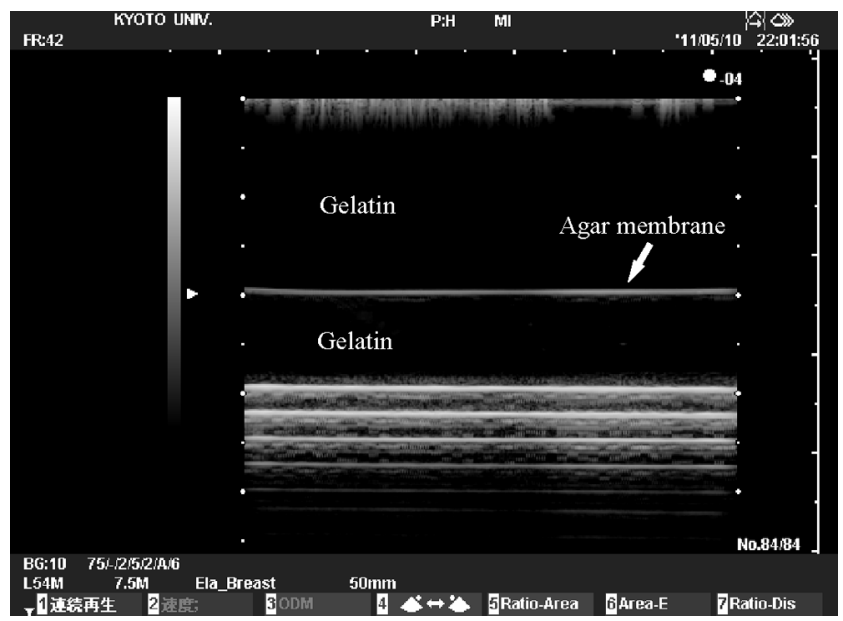

Fig. 13. B-mode image of a gelatin block with a $0.17-\mathrm{mm}$-thick agar membrane at a depth of $2 \mathrm{~cm}$, where the range interval is $0.05 \mathrm{~mm}$.

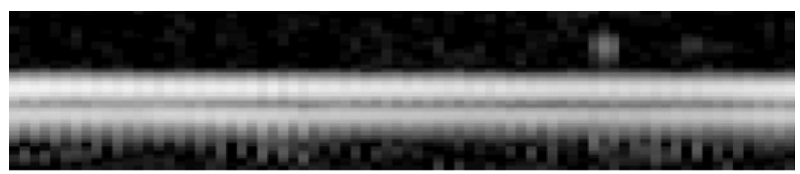

(a)

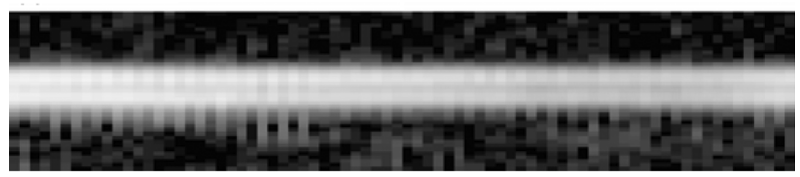

(b)

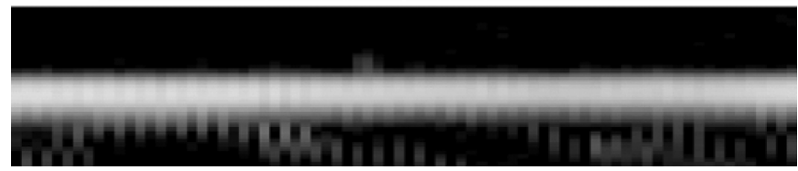

(c)

Fig. 14. B-mode images of agar membranes (a) 0.33 (b) 0.25 , and (c) $0.17 \mathrm{~mm}$ thick, where the size of ROIs is $2 \times 10 \mathrm{~mm}$, where the range interval is $0.05 \mathrm{~mm}$.

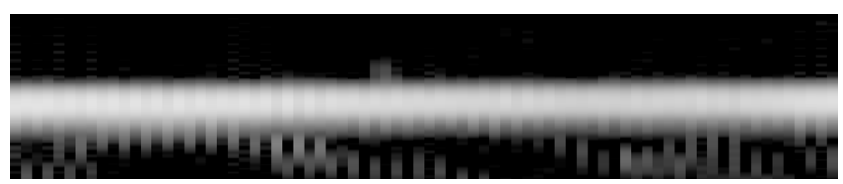

Fig. 15. Interpolated B-mode image of a $0.17-\mathrm{mm}$-thick agar membrane, where the size of ROIs is $2 \times 10 \mathrm{~mm}$ and the range interval is $0.01 \mathrm{~mm}$.

agar membrane that was $0.17 \mathrm{~mm}$ thick. Fig. 14 shows the enlarged B-mode images of agar membranes that were $0.33,0.25$, and $0.17 \mathrm{~mm}$ thick, where the estimated intensity of a conventional method was calculated from the scan line of IQ data. Fig. 15 shows the interpolated B-mode image of an agar membrane that was $0.17 \mathrm{~mm}$ thick, where the range interval of the image was adjusted to $0.01 \mathrm{~mm}$ using the zero-padding process. Since the range beam width of the B-mode image was wider than $0.05 \mathrm{~mm}$, the interpolation process had little effect in improving the image quality of the B-mode image.

For the improvement of the SNR of the received data, we coherently integrated five frames of data. Fig. 16 shows the 


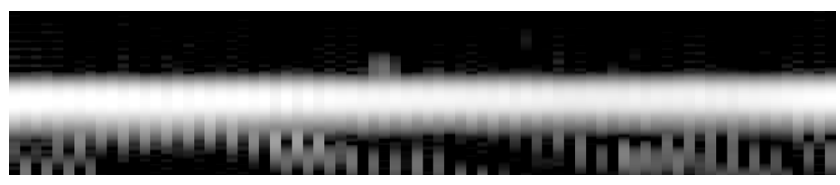

Fig. 16. Interpolated B-mode image of a 0.17 -mm-thick agar membrane using coherent integration, where the size of ROIs is $2 \times 10 \mathrm{~mm}$ and the range interval is $0.01 \mathrm{~mm}$. The number of coherent integration is five.

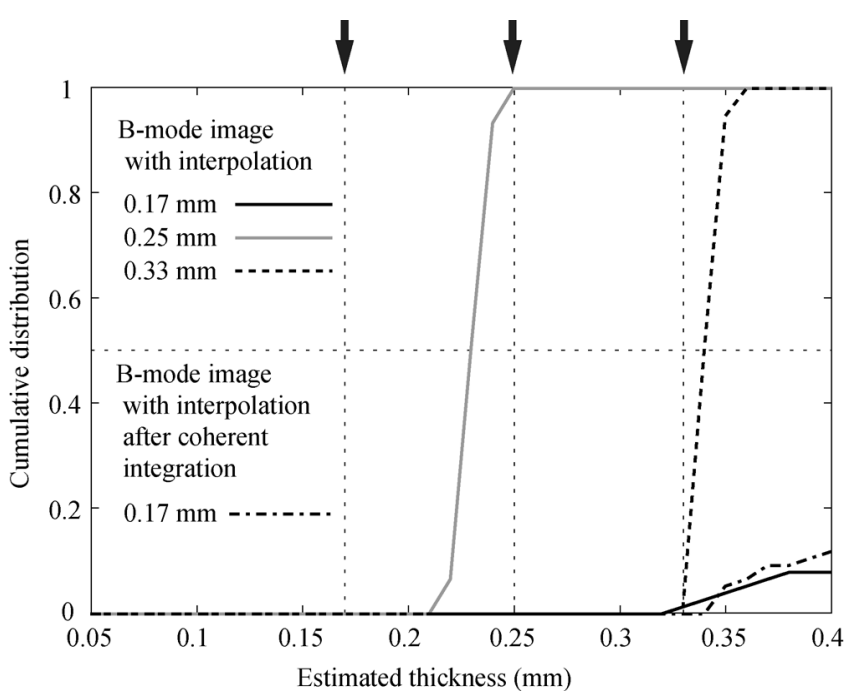

Fig. 17. Cumulative distributions of agar membrane thicknesses estimated using the B-mode images, where the range interval of the B-mode images was adjusted to $0.01 \mathrm{~mm}$ using interpolation. Arrows indicate the true thicknesses of agar membranes. The B-mode image of a $0.1 \mathrm{~mm}$ agar membrane with coherent integration was constructed from five frames of IQ data.

B-mode image of a 0.17 -mm-thick agar membrane using coherent integration, where the range interval was $0.01 \mathrm{~mm}$. Under the investigated condition, the improvement of the SNR of the received data hardly affected the image quality of the B-mode image.

We calculate the agar membrane thicknesses estimated by the conventional B-mode imaging method after the adjustment of the range interval to $0.01 \mathrm{~mm}$. We select the point with the highest echo intensity and the point with the second highest echo intensity from local maximum points in each scan line of the ROI, where the size of the ROI is $2 \times 10 \mathrm{~mm}$. The distance between the two selected positions is the estimated agar membrane thickness in the scan line. Fig. 17 shows the cumulative distribution of agar membrane thicknesses estimated using B-mode images with a $0.01 \mathrm{~mm}$ range interval. The median of the estimated thicknesses of a 0.33 -mm-thick agar membrane was $0.34 \mathrm{~mm}$, indicating the validity of the experimental study. The median of the estimated thicknesses of a 0.25 -mm-thick agar membrane was $0.23 \mathrm{~mm}$; however, the range resolution of the B-mode image was about $0.3 \mathrm{~mm}$, and caused the interference between the anterior and posterior boundaries, as shown in Fig. 14(b). In both cases, with and without coherent integration, conventional B-mode imaging after adjustment of the range interval to $0.01 \mathrm{~mm}$ failed to detect the couple of boundaries between the $0.17-\mathrm{mm}$-thick agar membrane and gelatin.

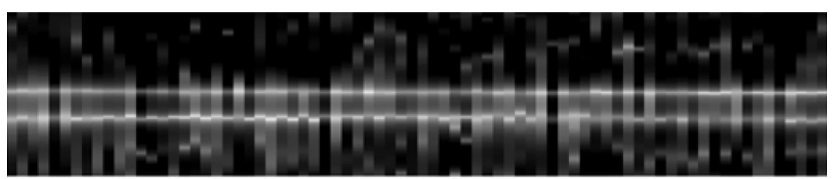

(a)

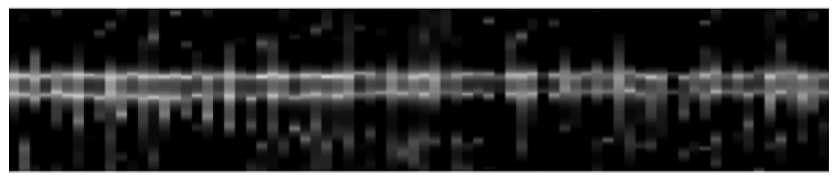

(b)

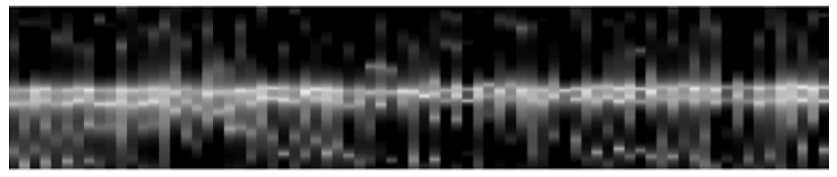

(c)

Fig. 18. FDI images of agar membranes (a) 0.33 (b) 0.25 , and (c) $0.17 \mathrm{~mm}$ thick, where the size of the ROIs is $2 \times 10 \mathrm{~mm}$.

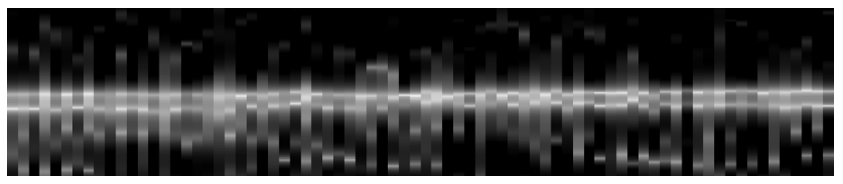

Fig. 19. FDI image of a 0.17 -mm-thick agar membrane using coherent integration, where the size of ROIs is $2 \times 10 \mathrm{~mm}$. The number of coherent integration is five.

Fig. 18 shows the images of the agar membranes $0.33,0.25$, and $0.17 \mathrm{~mm}$ thick obtained with the proposed FDI method. The proposed method used the same IQ data as the conventional B-mode imaging method. The FDI images of agar membranes 0.33 and $0.25 \mathrm{~mm}$ thick had narrower range beam widths than those of the B-mode images. In addition, the proposed method succeeded in depicting the couple of boundaries between the $0.17-\mathrm{mm}$-thick agar membrane and gelatin. Next, we coherently integrated five frames of IQ data to improve the SNR of the received data. Fig. 19 shows the FDI images of a 0.17 -mm-thick agar membrane after coherent integration using five frames.

Fig. 20 shows the cumulative distribution of agar membrane thicknesses estimated using FDI images. The medians of the estimated thicknesses of agar membranes that were $0.33,0.25$, and $0.17 \mathrm{~mm}$ thick were $0.31,0.23$, and $0.15 \mathrm{~mm}$, respectively. This result indicates that the proposed method has a range resolution less than $0.17 \mathrm{~mm}$. After the coherent integration using five frames of IQ data, the median of the estimated thicknesses of a 0.17 -mm-thick agar membrane became $0.16 \mathrm{~mm}$, and the distribution of the estimated thicknesses approached that of the true thicknesses. Since the increase in the SNR improves the range resolution of the FDI imager with the Capon method, this finding is consistent with a previous study [31].

Experiments were conducted using a commercial US device, but it was difficult to acquire the information concerning the setting of the time gain compensation from the manufacturer. However, the ROI was located at a depth of $20.5-21.5 \mathrm{~mm}$, indicating that the setting of the time gain compensation gave only a small influence to the result. 


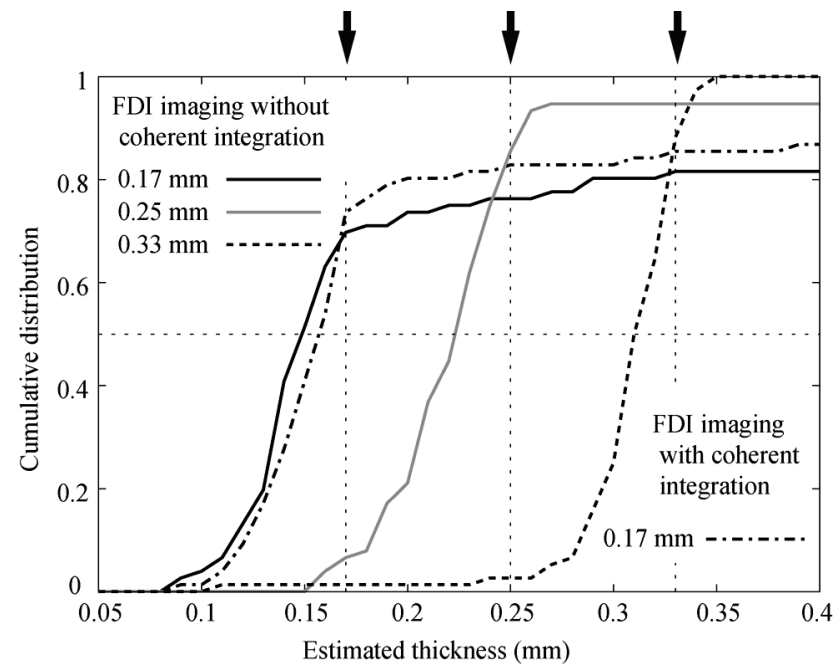

Fig. 20. Cumulative distributions of agar membrane thicknesses estimated using the images acquired using the proposed method, where the range interval of the images is $0.01 \mathrm{~mm}$. Arrows indicate the true thicknesses of agar membranes.

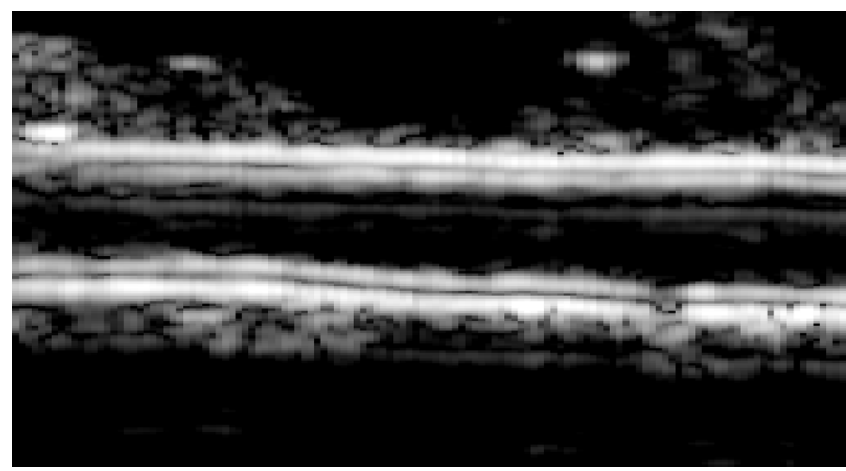

Fig. 21. Conventional B-mode image of a longitudinal section of a fresh swine femoral artery, where the size of the region of interest is $1 \times 1.67 \mathrm{~cm}$.

\section{Application of the Proposed Method to a Swine Femoral Artery}

Fig. 21 shows a B-mode image of a fresh swine femoral artery calculated from a frame of IQ data, where the size of the ROI was $1 \times 1.67 \mathrm{~cm}$. The estimated intensity calculated from the same frame of IQ data using the proposed method without the moving average is shown in Fig. 22. The image depicted by the proposed method has much higher range resolution than that of a B-mode image. The multiple scattering in a vessel wall causes the appearance of false boundaries after the true vessel wall boundaries. Therefore, the false boundaries appear after the anterior and posterior vessel wall boundaries in both B-mode and FDI images, as shown in Figs. 21 and 22. Fig. 23 shows the estimated intensity of the same frame using the proposed method with the moving average. This result suggests that the moving average improves the continuity of the acquired image in the lateral direction, at the cost of some range resolution. The average and standard deviation of the half-power widths of the posterior artery wall boundaries in the FDI image with the moving average were 0.054 and $0.025 \mathrm{~mm}$, respectively. In contrast, those in the B-mode image without the moving average were 0.182 and $0.025 \mathrm{~mm}$, respectively. In this evaluation, we

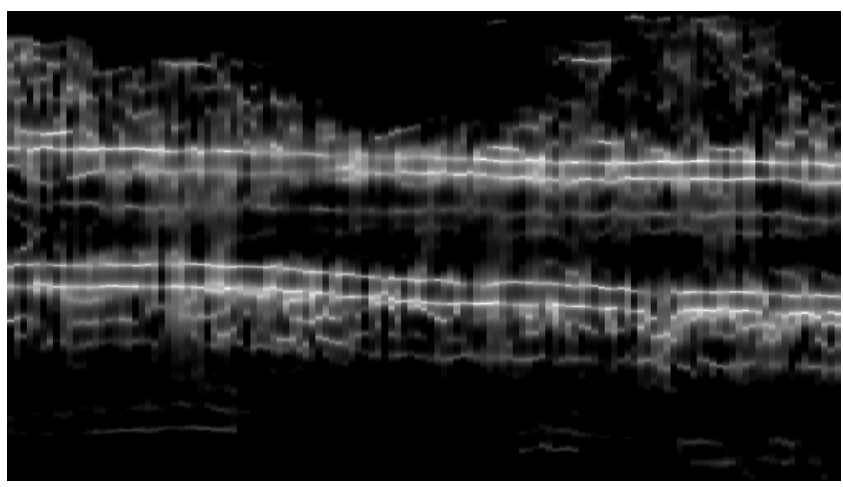

Fig. 22. Estimated intensity of a longitudinal section of a fresh swine femoral artery using the proposed method, where the size of region of interest is $1 \times$ $1.67 \mathrm{~cm}$.

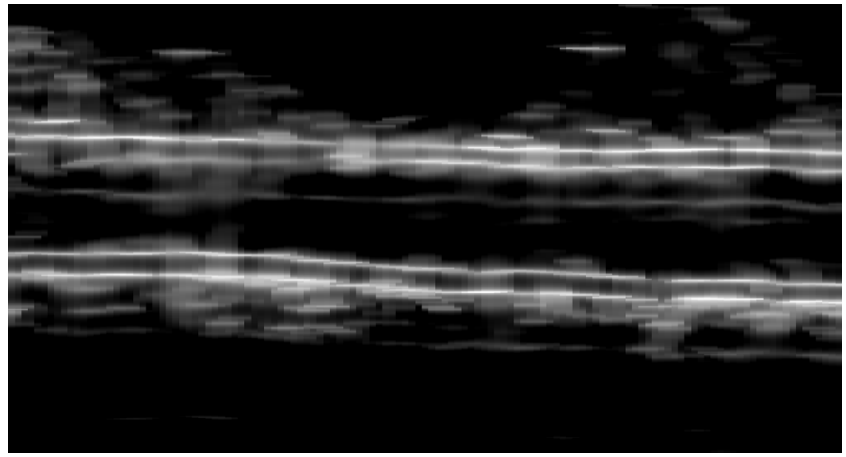

Fig. 23. Estimated intensity of a longitudinal section of a fresh swine femoral artery using the proposed method with the moving average in the lateral direction, where the size of region of interest is $1 \times 1.67 \mathrm{~cm}$.

investigated the posterior vessel wall boundaries in the $5-\mathrm{mm}$ wide center region of Figs. 21 and 23, i.e., 78 boundaries in 39 scan lines.

The thickness of the posterior wall of a carotid artery is often measured in order to detect artery thickening. To evaluate the performance of the proposed method in measuring vessel wall thickness, we investigated the differences in the posterior wall boundaries of a fixed femoral artery acquired by the FDI image and the conventional B-mode image from the true boundaries. As shown in Fig. 24, the true boundaries were traced onto a microscopic image by an anatomist. We selected the range of the vessel wall boundary couple in each scan line of the FDI image and the B-mode image using the following process. First, we selected the range of the maximum estimated intensity, $R_{1}$, in a scan line. Next, we chose the range of the maximum estimated intensity, $R_{2}$, under the constraint

$$
\left|R_{1}-R_{2}\right| \geq 0.2 \mathrm{~mm} \text {. }
$$

We employed these two peaks as the estimated boundaries of the vessel wall in the scan line. Since the $-3 \mathrm{~dB}$ width of the received signal returned from a single target is $0.2 \mathrm{~mm}$, the application of a constraint of less than $0.2 \mathrm{~mm}$ to a B-mode image causes the extraction of multiple peaks in a main lobe of the echo intensity returned from a single boundary. Therefore, the constraint in (34) should be no less than $0.2 \mathrm{~mm}$ for B-mode images. In contrast, the proposed method can employ a smaller 


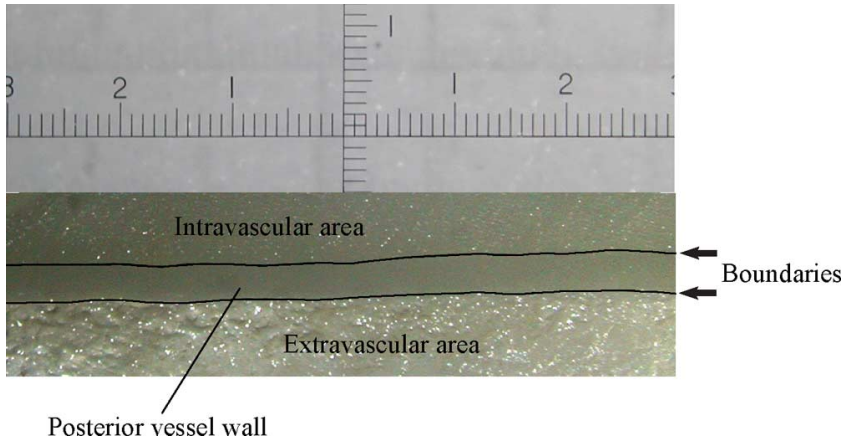

Fig. 24. Posterior wall boundaries of a fixed swine femoral artery traced by an anatomist onto a microscopic image with a millimeter scale.

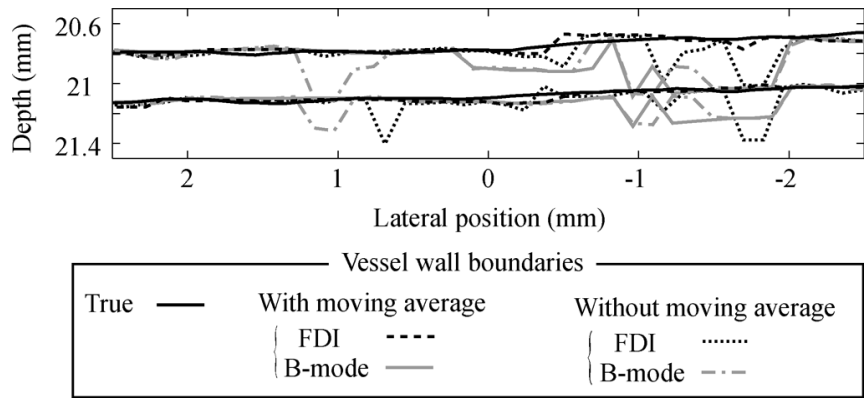

Fig. 25. Vessel wall boundaries of a fixed swine femoral artery embedded in an ager gel block. The FDI and B-mode boundaries were depicted by an image acquired using FDI and a B-mode image, respectively. The images with moving average employed the moving average in the lateral direction. The range interval of B-mode images is adjusted to $0.01 \mathrm{~mm}$ using interpolation.

constraint because the beam width of the proposed method is much narrower than that of the B-mode imaging method. To avoid the condition unfavorable to the conventional B-mode imaging method we chose the $0.2 \mathrm{~mm}$ constraint. When a ROI has a single peak in a scan line, the vessel wall boundaries depicted by the process show the wall thickness to be $0.2 \mathrm{~mm}$.

In this evaluation, we apply a moving average of five scan lines to B-mode images in the lateral direction. We separately adjust the depicted boundary couples using a FDI image and a B-mode image to the true boundary couple using a method of least squares. Fig. 25 shows the true boundary couple and the boundary couples depicted using a FDI image and a B-mode image after the position adjustment, where the images with moving average employ the moving average in the lateral direction. As shown in Fig. 25, the moving average in the lateral direction improves the continuity of the image in the lateral direction, suppressing the rupture of the estimated vessel wall boundaries for both FDI and B-mode imaging methods. Either the proposed and conventional method does not require the moving average, as shown in Figs. 21 and 22. To support the automatic detection of the vessel wall boundaries, we employ the moving average for both FDI and B-mode images in the estimation of vessel wall boundaries. The vessel wall boundary couple depicted using a FDI image are smooth and close to the true boundary couple in all scan lines. In contrast, the boundary couple depicted using a B-mode image with moving average are rough and swerve in several scan lines. The cumulative distributions of the distances, between true vessel

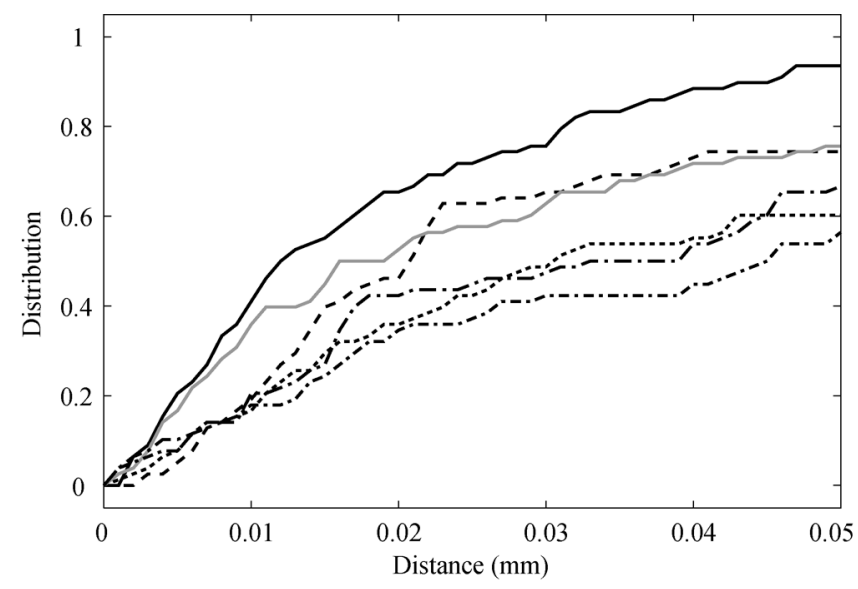

\begin{tabular}{|c|c|c|c|}
\hline FDI image & & B-mode imas & \\
\hline No additive noise & & No additive noise & 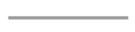 \\
\hline $\mathrm{SNR}=10 \mathrm{~dB}$ & ------ & $\mathrm{SNR}=10 \mathrm{~dB}$ & $-\cdot-\cdot-\cdot$ \\
\hline $\mathrm{SNR}=5 \mathrm{~dB}$ & (n) & $\mathrm{SNR}=5 \mathrm{~dB}$ & 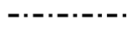 \\
\hline
\end{tabular}

(a)

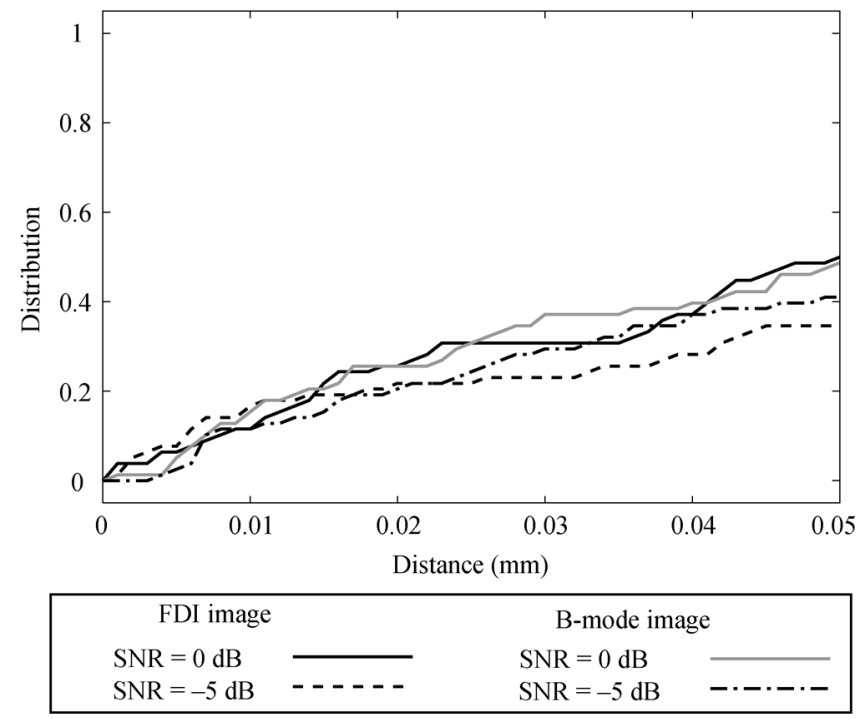

(b)

Fig. 26. Cumulative distribution of the distance between the true vessel wall boundaries and the estimated boundaries of a fixed femoral artery, where both images employed the moving average in the lateral direction. We used (a) the data without additive noise, and the data with additive white noise where the signal-to-noise ratio (SNR) was 10,5 (b) 0 and $-5 \mathrm{~dB}$. The range interval of B-mode images is adjusted to $0.01 \mathrm{~mm}$ using interpolation.

wall boundaries and the estimated boundaries using proposed FDI and B-mode images, are shown in Fig. 26 as the lines of no additive noise. Using a B-mode image with moving average, $57.7 \%$ of the estimated boundaries were located within 0.025 $\mathrm{mm}$ of the true boundaries. In contrast, using a FDI image $71.8 \%$ of the estimated boundary points were located within $0.025 \mathrm{~mm}$ of the true boundary. In all distances, the cumulative values of the proposed image were higher than those of the conventional B-mode image. This result indicates that the proposed method has the potential to improve the accuracy in vessel wall boundary depiction.

Next, we add white noise to the received data of a single frame, to examine the noise effect on the performance of the 


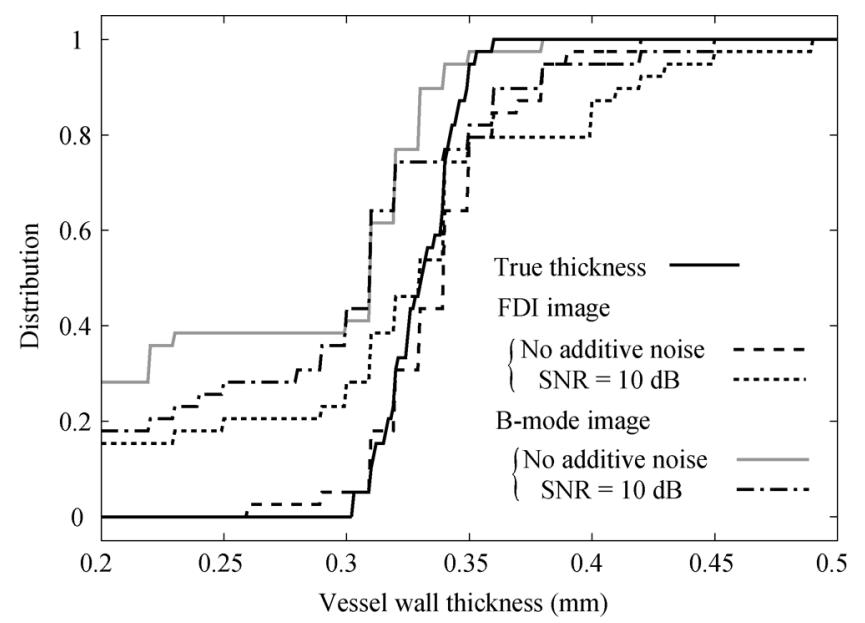

Fig. 27. The distribution of true artery wall thicknesses and the estimated thicknesses using the proposed method and a conventional method with the moving average in the lateral direction. The range interval of B-mode images is adjusted to $0.01 \mathrm{~mm}$ using interpolation.

proposed method. For the cases using the data with additive white noise, we employ the position adjustment parameters of the FDI and B-mode images without additive noise. The estimation of the vessel wall thickness needs to depict both of the two vessel wall boundaries. We thus define the SNR as the lower intensity of the two boundaries to the average noise intensity. Fig. 26 shows the cumulative distribution of the distance between the true vessel wall boundaries and the estimated boundaries of a fixed femoral artery. We used the data without additive noise and the data with additive white noise, where the SNR was $10,5,0$, and $-5 \mathrm{~dB}$. These results show that the proposed method has high potential in depicting vessel wall boundaries, as compared with a conventional method when SNR is $5 \mathrm{~dB}$ or higher. The decrease in SNR suppressed the performance of the proposed method in depicting vessel wall boundaries. However, the proposed method kept the performance equivalent to a conventional method. Fig. 27 shows the cumulative distribution of the true vessel wall thickness and the estimated vessel wall thickness calculated using the proposed images and B-mode images, where both images employ the moving average. Here, the estimated vessel wall thickness of $0.2 \mathrm{~mm}$ means that the boundary estimation failed to depict the two peaks in the scan lines. The proposed method succeeded in depicting two boundaries in $100 \%$ and $84.6 \%$ of the 39 scan lines in the $5-\mathrm{mm}$ wide region when there was no additive noise and SNR = $10 \mathrm{~dB}$, respectively. In contrast, the conventional method with the moving average succeeded in $71.8 \%$ and $82.1 \%$ of the same scan lines. The median of the true vessel wall thicknesses was $0.331 \mathrm{~mm}$. The medians of the estimated vessel wall thicknesses calculated using FDI images were 0.340 and $0.330 \mathrm{~mm}$ in the cases of no additive noise and $\mathrm{SNR}=10 \mathrm{~dB}$, respectively. In contrast, the medians of the estimated vessel wall thicknesses using B-mode images were both $0.310 \mathrm{~mm}$ in the same cases. Under the investigated condition, the results indicate that the proposed method has the potential to estimate the vessel wall thickness with an estimation error within $0.01 \mathrm{~mm}$.

\section{CONCLUSION}

In this paper, we propose a high range resolution imaging method suitable for US employing four techniques: frequency averaging, whitening, RF data oversampling and the moving average. The simulation study indicates that the proposed method has a range resolution of $0.05 \mathrm{~mm}$ in an ideal condition. The proposed method experimentally detected two boundaries that were $0.17 \mathrm{~mm}$ apart, using a single frame of IQ data acquired by a commercial US device. In contrast, a conventional method failed to distinguish the two boundaries from a single boundary. Using single frame data, the proposed method and the conventional method with the moving average in the lateral direction, depicted a swine femoral artery with half-power widths of 0.054 and $0.182 \mathrm{~mm}$ in the range direction, respectively. The estimation errors of the vessel wall thickness, using the proposed method and a conventional method with the moving average in the lateral direction, were 0.009 and $0.021 \mathrm{~mm}$, respectively. Throughout the experiment the proposed method employed the echo from the boundary between gelatin and agar as the reference signal. This implies that the proposed method works when the materials at the boundary for the acquisition of a reference signal are similar to those of the targets in the acoustic impedance and the sound velocity. The proposed method requires $7.7 \mathrm{~s}$ on a mobile PC with a single CPU for a $1 \times 3 \mathrm{~cm}$ ROI that consisted of 1001 range samples $\times 226$ scan lines. These results indicate the potential of the proposed method for the improvement of range resolution in ultrasonography without deterioration in temporal resolution, i.e., after signal processing of a minute using multiple CPUs we can acquire high range-resolution images without a decrease of the frame rate. Future works should include the image acquisition of clinical targets, e.g., human cervical artery.

\section{REFERENCES}

[1] E. G. Grant, C. B. Benson, G. L. Moneta, A. V. Alexandrov, J. D. Baker, E. I. Bluth, B. A. Carroll, M. Eliasziw, J. Gocke, B. S. Hertzberg, S. Katanick, L. Needleman, J. Pellerito, J. F. Polak, K. S. Rholl, D. L. Wooster, and R. E. Zierler, "Carotid artery stenosis: Gray-scale and Doppler US diagnosis - society of radiologists in ultrasound consensus conference," in Radiology, Nov. 2003, vol. 229, pp. 340-346.

[2] D. B. Hood, M. A. Mattos, A. Mansour, D. E. Ramsey, K. J. Hodgson, L. D. Barkmeier, and D. S. Sumner, "Prospective evaluation of new duplex criteria to indentify $70 \%$ internal carotid artery stenosis," J. Vasc. Surg., vol. 23, pp. 254-261, Feb. 1996.

[3] S. Byrd, P. Robless, A. Baxter, M. Emson, and A. Halliday, "Carotid duplex ultrasonography: Importance of standardization," Int. Angiol., vol. 17, pp. 248-254, Dec. 1998.

[4] E. G. Grant, A. J. Duerinckx, S. El Saden, M. L. Melany, G. Hathout, P. Zimmerman, S. N. Cohen, R. Singh, and J. D. Baker, "Doppler sonographic parameters for detection of carotid stenosis: Is there an optimum method for their selection?," AJR Am. J. Roentgnol., vol. 172, pp. 1123-1129, Apr. 1999.

[5] J. Huston, III, E. M. James, R. D. Brown, Jr, R. D. Lefsrud, D. M. I1strup, E. F. Robertson, F. B. Meyer, and J. W. Hallett, "Redefined duplex ultrasonographic criteria for diagnosis of carotid artery stenosis,' Proc. Mayo Clin., vol. 75, pp. 1133-1140, Nov. 2000.

[6] E. Kudeki and G. R. Stitt, "Frequency domain interferometry: A highresolution radar technique for studies at atmospheric turbulence," Geophys. Res. Lett., vol. 14, pp. 198-201, 1987.

[7] S. J. Franke, "Pulse compression and frequency domain interferometry with a frequency-hopped MST radar," Radio Sci., vol. 25, pp. 565-574, 1990.

[8] Y. H. Chu and T. Y. Chen, "Theoretical study of two-frequency coherence of MST radar returns,” Radio Sci., vol. 30, pp. 1803-1815, 1995. 
[9] H. Luce, M. Crochet, C. Hanuise, M. Yamamoto, and S. Fukao, "On the interpretation of the layered structures detected by MST radars in dual frequency domain interferometry (FDI) mode," Radio Sci., vol. 34, pp. 1077-1083, Sep. 1999.

[10] H. Luce, M. Yamamoto, S. Fukao, D. Helal, and M. Crochet, "A frequency domain radar interferometric imaging (FII) technique based on high-resolution methods," J. Atmos. Solar-Terr. Phys., vol. 63, pp. 221-234, Jan. 2001.

[11] J. Capon, "High resolution frequency-wavenumber spectrum analysis," Proc. IEEE, vol. 58, no. 8, pp. 1408-1418, Aug. 1969.

[12] O. L. Frost, "An algorithm for linear constrained adaptive array processing," Proc. IEEE, vol. 60, no. 8, pp. 926-935, Aug. 1972.

[13] W. F. Gabriel, "Spectral analysis and adaptive array super-resolution technique," Proc. IEEE, pp. 654-666, 1980.

[14] B. Widrow, K. M. Duvall, R. P. Gooch, and W. C. Newman, "Signal cancellation phenomena in adaptive antennas: Causes and cures," IEEE Trans. Antennas Propagat., vol. 30, no. 3, pp. 469-478, May 1982.

[15] T. J. Shan, M. Wax, and T. Kailath, "Spatial smoothing approach for location estimate of coherent sources," in Proc. 17th Asilomar Conf., 1983, pp. 367-371.

[16] T. J. Shan and T. Kailath, "Adaptive beamforming for coherent signals and interference," IEEE Trans. Acoust., Speech, Signal Process., vol. 33, no. 3, pp. 527-536, Jun. 1985.

[17] O. L. Frost, "Adaptive interference and multipath rejection in linear direction-finding array," Proc. ICASSP, pp. 2499-2502, 1986.

[18] K. Takao and N. Kikuma, "An adaptive array utilizing an adaptive spatial averaging technique for multipath environments," IEEE Trans. Antennas Propagat., vol. 35, no. 12, pp. 1389-1396, Dec. 1987.

[19] J. A. Mann and W. F. Walker, "A constrained adaptive beamformer for medical ultrasound: Initial results," in Proc. IEEE Ultrason. Symp., 2002, pp. 1807-1810

[20] J. A. Mann and W. F. Walker, "Constrained adaptive beamforming: Point and contrast resolution," Proc. SPIE, pp. 12-23, 2003.

[21] J. F. Synnevag, A. Austeng, and S. Holm, "Minimum variance adaptive beamforming applied to medical ultrasound imaging," in Proc. IEEE Ultrason. Symp., Sep. 2005, vol. 2, pp. 1199-1202.

[22] M. Sasso and C. Cohen-Barcie, "Medical ultrasound imaging using fully adaptive beamformer," Proc. Acoustics, Speech Signal Process., pp. 489-492, 2005.
[23] M. E. Ali and F. Schreib, "Adaptive single snapshot beamforming, a new concept for the rejection of nonstationary and coherent interferers," IEEE Trans. Signal Process., vol. 40, no. 12, pp. 3055-3058, Dec. 1992.

[24] R. Bethel, B. Shapo, and H. L. Van Trees, "Single snapshot spatial processing: Optimized and constrained," in Proc. Sensor Array Multichannel Signal Process. Workshop, 2002, pp. 508-512.

[25] F. Lingvall, "A method of improving overall resolution in ultrasonic array imaging using spatio-temporal deconvolution," Ultrasonics, vol. 422, pp. 961-968, Apr. 2004.

[26] B. M. Radich and K. M. Buckley, "Single-snapshot DOA estimation and source number detection," IEEE Trans. Signal Process., vol. 4, pp. 109-111, Apr. 1997.

[27] Y. Abe, H. Hasegawa, and H. Kanai, "Accurate estimation of scattering strength distribution by simultaneous reception of ultrasonic echoes with multichannel transducer array," Jpn. J. Appl. Phys., vol. 46, pp. 4813-4819, Jul. 2007.

[28] F. Viola, M. A. Ellis, and W. F. Walker, "Time-domain optimized nearfield estimator ultrasound imaging: Initial development and results," IEEE Trans. Med. Imag., vol. 27, no. 1, pp. 99-110, Jan. 2008.

[29] H. Taki, T. Kimura, T. Sakamoto, and T. Sato, "High resolution medical acoustic vascular imaging using frequency domain interferometry," Proc. IASTED Vis., Imag., Image Process., p. 652, 2009.

[30] H. Taki, K. Taki, T. Sakamoto, M. Yamakawa, T. Shiina, and T. Sato, "High range resolution medical acoustic vascular imaging with frequency domain interferometry," in Proc. IEEE EMBS, 2010, pp. $5298-5301$.

[31] L. Smaini, H. Luce, M. Crochet, and S. Fukao, "An improved high-resolution processing method for a frequency domain interferometric imaging (FII) technique," J. Atmos. Oceanic Technol., vol. 19, pp. 954-966, 2002.

[32] H. C. Andrews and B. R. Hunt, Digital image restoration. Upper Saddle River, NJ: Prentice Hall, 1977.

[33] A. Rosenfeld and A. C. Kak, Digital picture processing, 2nd ed. New York: Academic, 1982.

[34] R. C. Singleton, "An algorithm for computing the mixed radix fast Fourier transform," Proc. IEEE, vol. 17, no. 2, pp. 93-103, Jun. 1969. 